\title{
Robust IDA-PBC and PID-like Control for Port-Controlled Hamiltonian Systems
}

\author{
Mutaz Ryalat and Dina Shona Laila and Mohamed M. Torbati
}

\begin{abstract}
Interconnection and damping assignment passivity based control (IDA-PBC) is a method that has been developed to (asymptotically) stabilize nonlinear systems formulated in portcontrolled Hamiltonian (PCH) structure. This method has gained increasing popularity and has been successfully applied to a wide range of dynamical systems. However, little is known about the robustness of this method in response to the effects of uncertainty which could result from disturbances, noises, and modeling errors. This paper explores the possibility of extending the IDAPBC method by adopting a robustness perspective, with the aim of maintaining (asymptotic) stability of the system in the presence of such perturbations which exist in any realistic problem. We propose constructive results on Robust IDA-PBC and PID-like controllers for a class of $\mathrm{PCH}$ systems. The results extend some existing methods and provide a new framework that allows the implementation of integral action control to underactuated PCH systems that are quite commonly found in practice. The results are applied to a Quanser inertia wheel pendulum and illustrated through numerical simulations.
\end{abstract}

Index Terms-Hamiltonian systems, passivity-based control, integral control, adaptive control, robust control, input-to-state stability, underactuated systems.

\section{INTRODUCTION}

Control design methods for systems described by portcontrolled Hamiltonian (PCH) model have recently been investigated in several works (see [1] for a survey). Adopting the PCH structure that geometrically describes a large class of nonlinear models gives a number of advantages such as the obvious relation between the dynamics and the energy of the system, the energy conservative property that makes the model marginally stable to start with, and the coupling between the non-damping and the damping elements. However, this modeling approach results in exclusion of important ingredients of the system's dynamics such as the frictions. Hence, relying only on the pure PCH model, often results in a controller that works very well in simulation, but needs further adjustment in implementation [1], [2].

Besides the issue of modeling, complexity of systems and demand for control accuracy have made control design problems more challenging. System's perturbations such as, measurement noise, disturbances and model uncertainties are common problems that affect the performance of the control systems in industrial applications [2]. This motivates the establishment of the robust control paradigm, with the adaptive

Mutaz Ryalat, Dina Shona Laila and Mohamed Torbati are with the School of Engineering Sciences, Faculty of Engineering and the Environment, University of Southampton, Highfield, Southampton SO17 1BJ, United Kingdom. \{mmr3g11, d.laila, m.m.torbati\}@soton.ac.uk. M. Ryalat acknowledges the support of The German-Jordanian University (GJU)-Jordan for his PhD scholarship to carry out this research. and integral control among the main approaches. Broadly speaking, the integral action control is the most popular approach to deal with such effects and PID controller still dominates in practice.

The interconnection and damping assignment passivitybased control (IDA-PBC), introduced in [3], is a physically inspired control design method that invokes the principles of energy shaping and dissipation and formulated for systems described by PCH models. The main objective of this method is to stabilize the dynamical system by rendering the closedloop system passive (by shaping its energy) with a desired storage function (which is a proper Lyapunov function) [4]. Furthermore, the system can be asymptotically stabilized if it can be rendered strictly (output) passive by means of damping injection [5]. While IDA-PBC controller is theoretically proven to asymptotically stabilize classes of $\mathrm{PCH}$ systems; in real applications, the effect of disturbances, uncertainties or reference signal may deteriorate the performance of the control system [6], and the closed-loop system is more likely to suffer from steady-state errors or even instability. Apparently, when it comes to parametric uncertainties, the real-time implementation of control system requires a realtime and reasonably accurate estimate of these uncertainties. Thus, the main objective of this paper is to investigate the robust stabilization of perturbed $\mathrm{PCH}$ systems to encounter the effects of system's uncertainties.

Some solutions to deal with the robustness issue of $\mathrm{PCH}$ systems have been recently reported in [1], [6], [7]. In this paper, extension of results from [6], [7] for fully-actuated $\mathrm{PCH}$ mechanical systems are proposed. In Section [II-A we extend the robust PI controller of mechanical system proposed in [7] to the robust PID-like controller that provides a more general framework. In Section [II-B] inspired by the work of [6] and with a particular change of coordinates [7], we show that the integral action control can be incorporated to improve the robustness of IDA-PBC controller for PCH mechanical systems. Section III-C provides the most important contribution of the work reported in this paper, i.e. the integral control scheme for underactuated mechanical systems within PCH structure which has not been investigated in earlier literature. As we will discuss later, while often found in applications (see for instance the survey paper [8]), the system being underactuated significantly complicates the inclusion of the integral control. For this, we first introduce a technique to modify the structure of the model of the underactuated system, which then allows the implementation of the integral IDA controllers on the separable underactuated PCH systems, i.e PCH systems with constant inertia matrix. The robustness of the separable 
PCH mechanical systems under the presence of matched and unmatched time-varying disturbances is discussed in Section III-D. We show that using a certain change of coordinates a new method to characterize the property of system with disturbances such that the well-known input-to-state stability (ISS) property is satisfied. This method provides a simpler controller design than the one proposed in [7] on one hand. On the other hand, it provides a framework to apply this method to underactuated PCH systems. Furthermore, we also show that in some conditions asymptotic stability property of the closedloop system can also be achieved. Subsection III-E describes the design of a novel adaptive controller for uncertain PCH systems. Extension of all previous results to the case of nonseparable PCH system i.e. a system with non-constant inertia matrix is presented in Section IV] This results in more complex design as we need to take into account the derivative of the inertia matrices $M, M_{d}$. Finally, the results are validated in Section $\nabla$ where we apply our various proposed methods to robustly control an inertia wheel pendulum (IWP) system.

\section{Preliminaries}

The set of real and natural numbers (including 0) are denoted respectively by $\mathbb{R}$ and $\mathbb{N}$. Given an arbitrary matrix $G$, we denote the transpose and the pseudo inverse of $G$ by $G^{\top}$ and $G^{+}$, respectively. $G^{\perp}$ denotes the full rank left annihilator of $G$, i.e. $G^{\perp} G=0$. We denote an $n \times n$ identity matrix with $I_{n}$. For a vector $x \in \mathbb{R}^{n}$ and a matrix $A \in \mathbb{R}^{n \times n}$, we denote the Euclidean norm as $|x|$ and $|A|$, respectively and we denote the weighted norm $|x|_{A}:=x^{\top} A x$. For any continuous function $H(i, j)$, we define the gradient $\nabla_{i} H(i, j):=\partial H(i, j) / \partial i$. We use Young's inequality $\zeta \eta|y||z| \leq \frac{\zeta^{2}}{2}|y|^{2}+\frac{\eta^{2}}{2}|z|^{2}$ with positive constants $\zeta$ and $\eta$. We use a standard stability and passivity definitions for nonlinear systems [2]. Due to space limit, the arguments of functions are often dropped whenever they are clear from the context.

\section{A. Port-Controlled Hamiltonian Systems}

Consider a standard mechanical system whose dynamics are represented in a Port-Controlled Hamiltonian (PCH) form:

$$
\begin{aligned}
{\left[\begin{array}{c}
\dot{q} \\
\dot{p}
\end{array}\right] } & =\left[\begin{array}{cc}
0 & I_{n} \\
-I_{n} & 0
\end{array}\right]\left[\begin{array}{c}
\nabla_{q} H \\
\nabla_{p} H
\end{array}\right]+\left[\begin{array}{c}
0 \\
G(q)
\end{array}\right] u \\
y & =G^{\top}(q) \nabla_{p} H
\end{aligned}
$$

where $q, p \in \mathbb{R}^{n}$ are the states, $u$ and $y \in \mathbb{R}^{m}, m \leq n$, are the input and output variables, respectively. If $m=n$ the system is called fully-actuated, whereas if $m<n$ it is called underactuated. The Hamiltonian function, which is the total energy of the system, is defined as the sum of the kinetic energy and the potential energy

$$
H(q, p)=K(q, p)+V(q)=\frac{1}{2} p^{\top} M^{-1}(q) p+V(q),
$$

where $M(q)>0$ is the symmetric inertia matrix and $V(q)$ is the potential energy function. The PCH system is called separable if $M$ is constant, or otherwise it is called nonseparable. In PHC framework, the states $p$ and $q$ are known as the passive outputs and the non-passive outputs, respectively [6].

\section{B. Review on IDA-PBC Design}

We briefly review the general procedure of the IDA-PBC design as has been proposed for instance in [1], [3], [9]. Given a PCH system (1), by applying the IDA-PBC design we obtain the following preserved $\mathrm{PCH}$ dynamics

$$
\begin{aligned}
{\left[\begin{array}{c}
\dot{q} \\
\dot{p}
\end{array}\right] } & =\left[\begin{array}{cc}
0 & M^{-1} M_{d} \\
-M_{d} M^{-1} & J_{2}-R_{d}
\end{array}\right]\left[\begin{array}{c}
\nabla_{q} H_{d} \\
\nabla_{p} H_{d}
\end{array}\right] \\
y_{d} & =G^{\top}(q) \nabla_{p} H_{d},
\end{aligned}
$$

where

$$
H_{d}=\frac{1}{2} p^{\top} M_{d}^{-1}(q) p+V_{d}(q)
$$

is the desired total energy with

$$
\begin{gathered}
q_{e}=\arg \min H_{d}(q)=\arg \min V_{d}(q), \text { i.e. } \\
\nabla_{q} V_{d}\left(q_{e}\right)=0, \nabla_{q}^{2} V_{d}\left(q_{e}\right)>0,
\end{gathered}
$$

and $M_{d}=M_{d}^{\top}>0$ is the desired inertia matrix, $J_{2}=-J_{2}^{\top}$ is a free parameter, $R_{d}=G K_{v} G^{\top}>0$ is the dissipation (damping) matrix and $q_{e}$ is the equilibrium point to be stabilized. The system (3) is equivalent to the $\mathrm{PCH}$ system (1) with

$$
u_{I D A}=u_{e s}+u_{d i},
$$

with the energy shaping controller

$$
\begin{aligned}
u_{e s} & =\left(G^{\top} G\right)^{-1} G^{\top}\left(\nabla_{q} H-M_{d} M^{-1} \nabla_{q} H_{d}+J_{2} M_{d}^{-1} p\right) \\
& =G^{+}\left(\nabla_{q} H-M_{d} M^{-1} \nabla_{q} H_{d}+J_{2} M_{d}^{-1} p\right),
\end{aligned}
$$

and the damping injection (dissipation) controller

$$
u_{d i}=-K_{v} G^{\top} \nabla_{p} H_{d}, \quad K_{v}>0 .
$$

\section{Review on Integral Control (IC) within PCH Framework}

To improve control performance and robustness, particularly with respect to steady state error and reference inputs, the idea of applying integral action on the passive outputs of PCH systems has been proposed in [1]. It is well known that the integral action has the effect of eliminating offset. In this method the IC

$$
v=\int y_{d} d t=-K_{i} G^{\top} \int \nabla_{p} H_{d},
$$

with the integral gain $K_{i}=K_{i}^{\top}>0$, is added to the IDAPBC stabilized PCH system (3) to form an extended dynamical system

$$
\left[\begin{array}{c}
\dot{q} \\
\dot{p}
\end{array}\right]=\left[\begin{array}{cc}
0 & M^{-1} M_{d} \\
-M_{d} M^{-1} & J_{2}-R_{d}
\end{array}\right]\left[\begin{array}{c}
\nabla_{q} H_{d} \\
\nabla_{p} H_{d}
\end{array}\right]+\left[\begin{array}{l}
0 \\
G
\end{array}\right] v
$$

which can be written into an extended $\mathrm{PCH}$ form as

$$
\left[\begin{array}{c}
\dot{q} \\
\dot{p} \\
\dot{v}
\end{array}\right]=\left[\begin{array}{ccc}
0 & M^{-1} M_{d} & 0 \\
-M_{d} M^{-1} & J_{2}-R_{d} & G K_{i} \\
0 & -K_{i} G^{\top} & 0
\end{array}\right]\left[\begin{array}{c}
\nabla_{q} H_{d v} \\
\nabla_{p} H_{d v} \\
\nabla_{v} H_{d v}
\end{array}\right]
$$

where

$$
H_{d v}=H_{d}+\frac{1}{2} v^{\top} K_{i}^{-1} v .
$$

In reality, applying the IC only to the states which are the passive outputs is often insufficient. In Section [II, we present the extension of this approach to more general classes of $\mathrm{PCH}$ systems allowing the IC input on the states which are the nonpassive outputs. 


\section{Robust Control of Separable Hamiltonian SYSTEMS}

Subsections $\amalg I-A$ and $\amalg I-B$ represent extensions to the results in [6] and [7] which deal with the fully-actuated mechanical systems. Then, subsection $\amalg I I-C$ extends the integral IDA-PBC results to deal with underactuated mechanical systems.

\section{A. PID-like Control for Separable PCH Systems}

In [7], a PI controller has been proposed to reject constant disturbance(s) for the case of a separable PCH system which is assumed to have natural damping. On the contrary, here we start with assuming that the system does not have natural damping and we introduce the damping to the system (1) by means of a derivative controller, thus, we obtain the PID-like controller. This assumption of no damping is consistent with the PCH model that we consider in this paper.

Remark 3.1: Note that we call the controller as PID-like controller because it consists of the P, I and D terms. However, this controller is a state feedback controller and not exactly the same as the conventional PID controller which sits on the feed-forward path of the system. This type of control has been used for instance in [7].

Proposition 3.1: Consider the separable (and fully-actuated) PCH system (1). Define the state transformation

$$
x_{q}=q ; \quad x_{p}=p+M G x_{v}
$$

to realize the closed-loop system in the new variables $x:=$ $\left[x_{q} x_{p} x_{v}\right]$ as

$$
\left[\begin{array}{c}
\dot{x}_{q} \\
\dot{x}_{p} \\
\dot{x}_{v}
\end{array}\right]=\left[\begin{array}{ccc}
0 & I_{n} & -G K_{i} \\
-I_{n} & -K_{d} & 0 \\
K_{i} G^{\top} & 0 & 0
\end{array}\right]\left[\begin{array}{c}
\nabla_{x_{q}} H_{x} \\
\nabla_{x_{p}} H_{x} \\
\nabla_{x_{v}} H_{x}
\end{array}\right],
$$

with the Hamiltonian function

$$
H_{x}=\frac{1}{2} x_{p}^{\top} M^{-1} x_{p}+\frac{1}{2} x_{v}^{\top} K_{i}^{-1} x_{v}+V\left(x_{q}\right) .
$$

Then, the PID-like controller

$$
\begin{aligned}
u= & -M \dot{x}_{v}-K_{d} x_{v}-K_{d} M^{-1} p \\
= & -\underbrace{M K_{i} G^{\top} \nabla_{x_{q}} V\left(x_{q}\right)}_{\text {Proportional }}-\underbrace{K_{d} K_{i} G^{\top} \int \nabla_{x_{q}} V\left(x_{q}\right) d t}_{\text {Integral }} \\
& -\underbrace{K_{d} M^{-1} p}_{\text {Derivative }}, \quad\left(\dot{q}=M^{-1} p\right)
\end{aligned}
$$

where $K_{i}=K_{i}^{\top}>0, K_{d}=K_{d}^{\top}>0$, is an asymptotically stabilizing controller for the system.

Proof of Proposition 3.1: Consider the Hamiltonian function (15) as a candidate Lyapunov function for the system (14). Because $M$ is constant, then $\nabla_{x_{q}} H_{x}=\nabla_{x_{q}} V$. Its derivative along the trajectories of the system is

$$
\begin{aligned}
\dot{H}_{x}= & x_{p}^{\top} M^{-1} \dot{x}_{p}+x_{v}^{\top} K_{i}^{-1} \dot{x}_{v}+\nabla V_{x_{q}}^{\top}\left(x_{q}\right) \dot{x}_{q} \\
= & -x_{p}^{\top} M^{-1} \nabla V_{x_{q}}-x_{p}^{\top} M^{-1} K_{d} M^{-1} x_{p} \\
& +x_{v}^{\top} K_{i}^{-1}\left(K_{i} G^{\top} \nabla V_{x_{q}}\right)+\nabla V_{x_{q}}^{\top}\left(x_{p}^{\top} M^{-1}-G x_{v}\right) \\
= & -x_{p}^{\top} M^{-1} K_{d} M^{-1} x_{p},
\end{aligned}
$$

which is negative semi-definite. By invoking LaSalle's invariance principle [2], one can prove that the largest invariant set contained in $\Omega=\left\{x_{\{q, p, v\}}: \dot{H}_{x}=-x_{p}^{\top} M^{-1} K_{d} M^{-1} x_{p}=\right.$ $\left.0 \mid x_{p}=0\right\}$ is the equilibrium point $x_{e}=\left(x_{q e}, 0,0\right)=$ $\left(q_{e}, 0,0\right)$, thus it is asymptotically stable (see the proof of Proposition 3.2). The PID-like controller (16) is found by equating (11) and (14) and applying the coordinate transformation (13), that is

$$
\begin{aligned}
\dot{p} & \equiv \dot{x}_{p}-M \dot{x}_{v} \\
-\nabla_{q} H+G u & =-\nabla_{x_{q}} H_{x}-K_{d} \nabla_{x_{p}} H_{x}-M \dot{x}_{v} .
\end{aligned}
$$

Notice that with (13) we have $\nabla_{q} H=\nabla_{x_{q}} H_{x}$ and $\nabla_{x_{p}} H_{x}=$ $M^{-1}\left(p+M x_{v}\right)$, thus, we obtain (16).

Remark 3.2: The PCH structure of the original model (1) has been preserved in the augmented system (14). This can be shown from i) the coincidence of the state equations of both models (they are matched) ii) the preservation of the Hamiltonian and the PCH structure [7] of the model, i.e. the positive definiteness of the interconnection matrix. This preservation in the closed-loop system ensures asymptotic stability of the system as shown in the Proof of Proposition 3.1 and robustness property is provided through the introduction of the integral action.

\section{B. Integral IDA-PBC for Separable PCH Systems}

In Section III-A a PID-like controller has been proposed for both asymptotically stabilizing and robustifying the fullyactuated PCH system. In this section, we assume that the stabilization problem has been solved using IDA-PBC method and we need to introduce an integral action to solve the robustness issue. As discussed in Section III-C, a method to include the IC for passive outputs has existed. However for non-passive outputs, it is difficult to add the IC action while preserving the $\mathrm{PCH}$ structure and stability properties simultaneously.

In [10] a method that involves canonical transformation of coordinates and solving a set of PDEs was proposed. Coordinate transformation was also used in [11] to deal with the robust control of non-passive outputs with unmatched disturbances. An initial result towards applying IC on nonpassive outputs of PCH systems has been recently proposed in [6]. In this method the IC is added to the $\mathrm{PCH}$ model that has already been stabilized using a PBC method, exploiting a state transformation that preserves the $\mathrm{PCH}$ structure of the open-loop system. However, this method requires solving a set of algebraic equations that account for defining the state transformation which makes it quite complicated.

Inspired by the work of [6], [7], [11], we present a simpler method to include the IC for non-passive outputs of PCH system assuming a stabilizing controller has already been obtained and we are dealing with steady-state error. The main idea is to use the change of coordinates as in Section III-A and [7] to obtain the IC, while preserving the structure and stability properties of the original PCH model.

Consider the closed-loop PCH system (10) with equilibrium satisfying (5) when $v=0$. Since throughout the IDA-PBC 
design procedures, $J_{2}$ is set to 0 as both $M$ and $M_{d}$ are constants [12], the system can be rewritten as

$$
\left[\begin{array}{c}
\dot{q} \\
\dot{p}
\end{array}\right]=\left[\begin{array}{cc}
0 & M^{-1} M_{d} \\
-M_{d} M^{-1} & -R_{d}
\end{array}\right]\left[\begin{array}{c}
\nabla_{q} H_{d} \\
\nabla_{p} H_{d}
\end{array}\right]+\left[\begin{array}{c}
0 \\
G
\end{array}\right] v,
$$

Proposition 3.2: Consider the separable PCH system (1). Assume a stabilizing IDA-PBC controller (6) has already been obtained with the desired Hamiltonian (4) and the desired PCH dynamics take the form (17). Defining the state transformation (13) to realize the augmented closed-loop PCH system

$$
\left[\begin{array}{c}
\dot{x}_{q} \\
\dot{x}_{p} \\
\dot{x}_{v}
\end{array}\right]=\left[\begin{array}{ccc}
0 & M^{-1} M_{d} & -G K_{i} \\
-M_{d} M^{-1} & -R_{d} & 0 \\
K_{i} G^{\top} & 0 & 0
\end{array}\right]\left[\begin{array}{c}
\nabla_{x_{q}} H_{x} \\
\nabla_{x_{p}} H_{x} \\
\nabla_{x_{v}} H_{x}
\end{array}\right]
$$

with

$$
H_{x}=\frac{1}{2} x_{p}^{\top} M_{d}^{-1} x_{p}+\frac{1}{2} x_{v}^{\top} K_{i}^{-1} x_{v}+V\left(x_{q}\right) .
$$

Asymptotic stability of the equilibrium point $x_{e}=$ $\left(x_{q e}, 0,0\right)=\left(q_{e}, 0,0\right)$ is preserved with the IC

$$
\begin{aligned}
v & =-R_{d} M_{d}^{-1} M G x_{v} \\
\dot{x}_{v} & =K_{i} G^{\top} \nabla_{x_{q}} H_{x} .
\end{aligned}
$$

Furthermore, the total control input with integral action takes the form

$$
u=u_{I D A}+v+u_{p}
$$

where $u_{p}=-M K_{i} G^{\top} \nabla_{x_{q}} V\left(x_{q}\right)$ is an additional control term that appears through the procedure of finding the closedloop controller.

Proof of Proposition [3.2; The proof can be established following the same procedures as in the proof of Proposition 3.1. Furthermore, in view of (5), (13) and (19), we obtain $\dot{H}_{x}=-x_{p}^{\top} M_{d}^{-1} R_{d} M_{d}^{-1} x_{p} \leq 0$, i.e the system is stable. LaSalle's invariance principle is then used to prove that the largest invariant set contained in $\Omega=\left\{x_{\{q, p, v\}}: \dot{H}_{x}=\right.$ $\left.-x_{p}^{\top} M_{d}^{-1} R_{d} M_{d}^{-1} x_{p}=0 \mid x_{p}=0\right\}$ is the equilibrium point $x_{e}=\left(q_{e}, 0,0\right)$, thus it is asymptotically stable. Notice that for the system to maintain $\dot{H}_{x}=0$ condition, the trajectory must be confined to $x_{p}=0$. Using the system closed-loop dynamics (18) we show that

$$
x_{p} \equiv 0 \Longrightarrow \dot{x}_{p} \equiv 0 \Longrightarrow \nabla_{x_{q}} H_{x}=0 .
$$

For PCH systems, the gradient of the potential energy function vanishes $\left(\nabla_{x_{q}} H_{x}=\nabla_{x_{q}} V\left(x_{q}\right)=0\right)$ if the system converges to its equilibrium point $q_{e}$ (see (5), Section IV in [13] and Lemma 4.2. in [14]). Thus,

$$
\nabla_{x_{q}} H_{x} \equiv 0 \Longrightarrow x_{q}=q_{e} \text { and } x_{v}=0 .
$$

Hence, the system can maintain $\dot{H}_{x}=0$ only at the equilibrium point $x_{e}=\left(q_{e}, 0,0\right)$, which proves that this equilibrium is asymptotically stable. The controller is obtained by matching the momenta of (17) and (18), that is

$$
\begin{aligned}
\dot{p} & =-M_{d} M^{-1} \nabla_{q} H_{d}-R_{d} \nabla_{p} H_{d}+v \\
& \equiv \dot{x}_{p}-M G \dot{x}_{v} \\
& =-M_{d} M^{-1} \nabla_{x_{q}} H_{x}-R_{d} \nabla_{x_{p}} H_{x}-M G \dot{x}_{v} .
\end{aligned}
$$

Solving (22), we get (20) and (21).
Remark 3.3: The IC laws (16) and (20) obtained in the PIDlike and IIDA methods, respectively, are very similar, except that there is the term $M_{d}^{-1} M$ in the one constructed using IIDA. This is due to the different interconnection matrices used for the design; the original interconnection matrix in the case of PID-like and the desired one in the case of IIDA. Also, the total control input $u$ of IIDA includes an additional proportional control term $u_{p}$.

\section{IC for Underactuated PCH Mechanical Systems}

In Subsections $\amalg I-A$ and $\amalg I-B$, we have discussed the construction of controllers for separable PCH systems, requiring the input matrix $G$ to be full rank. This condition makes the application to underactuated systems in which $G$ is non full rank, not straightforward, whereas these systems are often found in practice, either by design or due to faults. In this section, a more general result, the design of IC action for underactuated mechanical systems is proposed.

While PCH models allow some extensions in the system coordinates, such as adding integral action, two main properties must be ensured when these extensions are added to the model:

a. Preserving the PCH structure matrix (consult [15] for detailed formulation). The extension must not break the skew-symmetry of the interconnection matrix and the positive definiteness of the dissipation matrix.

b. Preserving the passivity and (asymptotic) stability of the closed-loop system.

Due to these constraints, all existing IC schemes within PCH framework were limited to fully-actuated mechanical systems, imposing the following conditions:

(i) The input matrix $G$ is full rank.

(ii) $G=G^{\top}$, or sometimes $K_{i}=K_{i}^{\top}$ is used instead.

In fact, Condition (i) is not necessary because the $\mathrm{PCH}$ structure can still be preserved even if the system is underactuated. This can be proved for instance using the Schur's complement [16], by showing the positive definiteness of the interconnection and dissipation matrices even if $\operatorname{rank}(G)=$ $m<n$. Moreover, the formalism of IDA-PBC for underactuated mechanical systems also shows that the $\mathrm{PCH}$ structure is preserved even when $G$ is not invertible [12].

Unfortunately, stability cannot be easily verified if the integral action is added to the underactuated PCH mechanical systems, because Condition (ii) is not satisfied. This can be illustrated in the following case. A simple calculation of the derivative of the Hamiltonian function (19) along the trajectories of the system gives

$$
\dot{H}_{x}=-x_{p}^{\top} M_{d}^{-1} R_{d} M_{d}^{-1} x_{p}-M_{d}^{-1} x_{p} G x_{v}+M_{d}^{-1} x_{p} G^{\top} x_{v} .
$$

If $G=G^{\top}$ like in the case of fully-actuated PCH system, the last two terms are equal except with opposite signs, thus cancel out each other. Hence,

$$
\dot{H}_{x}=-x_{p}^{\top} M_{d}^{-1} R_{d} M_{d}^{-1} x_{p} \leq 0,
$$

which proves the stability of $x_{e}$. For underactuated mechanical systems, since $G \in \mathbb{R}^{n \times m} \neq G^{\top} \in \mathbb{R}^{m \times n}$, we cannot 
draw any conclusion about the stability of the system. A similar illustration can also be shown for the IC presented in Section II-C. Therefore, modification is needed to deal with underactuated systems, as discussed next.

1) Integral control on passive outputs: Here, we present results for underactuated $\mathrm{PCH}$ systems with $n=2, m=1$. Recall the desired closed-loop PCH system (11). For underactuated systems, the matrix $G$ can be defined as

$$
G=\left[\begin{array}{l}
g_{1} \\
g_{2}
\end{array}\right] \quad\left(G^{\top}=\left[\begin{array}{ll}
g_{1} & g_{2}
\end{array}\right]\right) .
$$

Depending on how the input acts on the states, we may have either

$$
G_{1}=\left[\begin{array}{c}
g_{1} \\
0
\end{array}\right] \quad\left(G_{1}^{\top}=\left[\begin{array}{ll}
g_{1} & 0
\end{array}\right]\right),
$$

if the first passive output receives the direct action from the input, or

$$
G_{2}=\left[\begin{array}{c}
0 \\
g_{2}
\end{array}\right] \quad\left(G_{2}^{\top}=\left[\begin{array}{ll}
0 & g_{2}
\end{array}\right]\right),
$$

if the second passive output receives the direct action from the input. Instead, and without loss of generality, for the augmented system we define a new matrix $\mathcal{G}$ as

$$
\mathcal{G}=\left[\begin{array}{cc}
g_{1} & 0 \\
0 & g_{2}
\end{array}\right],\left(\mathcal{G}^{\top}=\left[\begin{array}{cc}
g_{1} & 0 \\
0 & g_{2}
\end{array}\right]\right)
$$

thus, we have either $g_{2}=0$ corresponding to $G_{1}$, or $g_{1}=0$ corresponding to $G_{2}$.

Remark 3.4: Notice that $\mathcal{G}=\mathcal{G}^{\top}$. When either $g_{1}$ or $g_{2}$ is zero, exclusively, replacing $G$ with $\mathcal{G}$ in (11) neither breaks the $\mathrm{PCH}$ structure (the dynamics) nor changes the contribution of the augmented state to the system. This is due to the fact that $\operatorname{rank}(G)=\operatorname{rank}(\mathcal{G})$. For example, using $G_{1}$ in the extended state equation, we have

$$
\begin{aligned}
\dot{v} & =-K_{i} G^{\top} \nabla_{p} H_{d v} \\
& =-K_{i}\left[\begin{array}{ll}
g_{1} & 0
\end{array}\right]\left[\begin{array}{c}
\nabla_{p_{1}} H_{d v} \\
\nabla_{p_{2}} H_{d v}
\end{array}\right]=-K_{i} g_{1} \nabla_{p_{1}} H_{d v},
\end{aligned}
$$

and using $\mathcal{G}_{1}$, we also obtain

$$
\begin{aligned}
\dot{v} & =-K_{i} \mathcal{G}_{1}^{\top} \nabla_{p} H_{d v} \\
& =-K_{i}\left[\begin{array}{cr}
g_{1} & 0 \\
0 & 0
\end{array}\right]\left[\begin{array}{c}
\nabla_{p_{1}} H_{d v} \\
\nabla_{p_{2}} H_{d v}
\end{array}\right]=\left[\begin{array}{c}
-K_{i} g_{1} \nabla_{p_{1}} H_{d v} \\
0
\end{array}\right] .
\end{aligned}
$$

As the last column is zero, this shows that the same result is obtained in both cases, and the PCH dynamics are preserved. The same case also applies to $\left(G_{2}, \mathcal{G}_{2}\right)$. Hence, with this substitution we obtain

$$
\dot{H}_{d v}=-p^{\top} M_{d}^{-1} R_{d} M_{d}^{-1} p \leq 0,
$$

which proves the stability of the system.

Remark 3.5: Note that the replacement of $G$ with $\mathcal{G}$, is not meant to change the input matrix of the original PCH system, but it is applied to the augmented system to proceed with the design procedures.

Proposition 3.3: Replacing the $G$ in the PCH model (11) with $\mathcal{G}$ to obtain

$$
\left[\begin{array}{c}
\dot{q} \\
\dot{p} \\
\dot{v}
\end{array}\right]=\left[\begin{array}{ccc}
0 & M^{-1} M_{d} & 0 \\
-M_{d} M^{-1} & J_{2}-R_{d} & \mathcal{G} K_{i} \\
0 & -K_{i} \mathcal{G}^{\top} & 0
\end{array}\right]\left[\begin{array}{c}
\nabla_{q} H_{d v} \\
\nabla_{p} H_{d v} \\
\nabla_{v} H_{d v}
\end{array}\right],
$$

allows for the integral action to be applied to underactuated mechanical systems.

Proof of Proposition 3.3 is established in Remark 3.4

2) Integral control on non-passive outputs: In Subsection III-C1 we have considered IC on passive outputs. However in the context of PCH mechanical systems, non-passive outputs, usually being the states representing displacement or positions, are often the outputs of interest. In this subsection we will present the method of introducing the integral action on nonpassive outputs for underactuated systems. The construction of this IC for non-passive outputs follows closely the procedures as in Section III-B, with the replacement of matrix $G$ by $\mathcal{G}$.

Introducing the IC action to the non-passive outputs, in the same way as how it was done to the passive outputs, yields the closed-loop PCH system (note: compare this with 25):

$$
\left[\begin{array}{c}
\dot{q} \\
\dot{p} \\
\dot{v}
\end{array}\right]=\left[\begin{array}{ccc}
0 & M^{-1} M_{d} & 0 \\
-M_{d} M^{-1} & J_{2}-R_{d} & \mathcal{G} K_{i} \\
-K_{i} \mathcal{G}^{\top} & 0 & 0
\end{array}\right]\left[\begin{array}{c}
\nabla_{q} H_{d v} \\
\nabla_{p} H_{d v} \\
\nabla_{v} H_{d v}
\end{array}\right] .
$$

However, this way destroys the $\mathrm{PCH}$ structure of the system (the system is no more Hamiltonian), which is obvious from the unsymmetrical interconnection matrix.

Another option is to write the augmented system as

$$
\left[\begin{array}{c}
\dot{q} \\
\dot{p} \\
\dot{v}
\end{array}\right]=\left[\begin{array}{ccc}
0 & M^{-1} M_{d} & \mathcal{G} K_{i} \\
-M_{d} M^{-1} & J_{2}-R_{d} & 0 \\
-K_{i} \mathcal{G}^{\top} & 0 & 0
\end{array}\right]\left[\begin{array}{c}
\nabla_{q} H_{d v} \\
\nabla_{p} H_{d v} \\
\nabla_{v} H_{d v}
\end{array}\right],
$$

which preserves the $\mathrm{PCH}$ structure. However, with this form, the IC action is not included in the control law, i.e. the IC term is not attainable from the augmented system. To solve this, the integral action is admitted by means of coordinates transformation as stated in the following proposition.

Proposition 3.4: Consider the separable PCH system (1) with $G$ non-full rank. Assume a stabilizing IDA-PBC controller (6) is already obtained with the desired (closed-loop) energy function (4) and the desired $\mathrm{PCH}$ dynamics take the form (17). We employ the state transformation (13) to realize the augmented closed-loop PCH system

$$
\left[\begin{array}{c}
\dot{x}_{q} \\
\dot{x}_{p} \\
\dot{x}_{v}
\end{array}\right]=\left[\begin{array}{ccc}
0 & M^{-1} M_{d} & -\mathcal{G} K_{i} \\
-M_{d} M^{-1} & -R_{d} & 0 \\
K_{i} \mathcal{G}^{\top} & 0 & 0
\end{array}\right]\left[\begin{array}{c}
\nabla_{x_{q}} H_{x} \\
\nabla_{x_{p}} H_{x} \\
\nabla_{x_{v}} H_{x}
\end{array}\right],
$$

with the Hamiltonian function (19) and replacing $G$ with $\mathcal{G}$. Then, asymptotic stability of the equilibrium point $x_{e}=$ $\left(q_{e}, 0,0\right)$ is preserved with the IC

$$
\begin{aligned}
v & =-G^{+} R_{d} M_{d}^{-1} M \mathcal{G} x_{v} \\
\dot{x}_{v} & =K_{i} \mathcal{G}^{\top} \nabla_{x_{q}} H_{x} .
\end{aligned}
$$

Furthermore, the total control input takes the form

$$
u=u_{I D A}+v+u_{p}
$$

where $u_{p}=-G^{+} M K_{i} \mathcal{G} \dot{x}_{v}$ is an additional control term which appears through the procedure of finding the feedback controller.

Proof of Proposition 3.4 is established following the same procedures as in the proof of Proposition 3.2 and involving Remark 3.4 .

In Section V we will show by example, how this IC action eliminates the steady-state error. 


\section{ISS for Separable PCH Systems using IDA-PBC method}

Here, we show our results for the input-to-state stability (ISS) control for separable PCH mechanical systems with time-varying disturbances employing IDA-PBC method to obtain the stabilizing controller. The theory of input-to-state stability (ISS) introduced in [17] is an extension of Lyapunov stability theory to deal with systems with inputs. ISS combines the Lyapunov stability notion and the bounded-input-boundedoutput (BIBO) stability notion [18]. ISS is a central tool in nonlinear systems analysis that studies the influence of inputs and disturbances on the system and the robustness of the system with respect to such inputs. Here, we use the definition of ISS as stated in [18, Section 3.3] and [19, Remark 2.4].

First, we will show our results for the fully-actuated mechanical systems, and then we show some extensions to deal with the under-actuation case. Consider the PCH system

$$
\left[\begin{array}{c}
\dot{q} \\
\dot{p}
\end{array}\right]=\left[\begin{array}{cc}
0 & I_{n} \\
-I_{n} & 0
\end{array}\right]\left[\begin{array}{c}
\nabla_{q} H \\
\nabla_{p} H
\end{array}\right]+\left[\begin{array}{l}
0 \\
G
\end{array}\right] u+\left[\begin{array}{l}
d_{1} \\
d_{2}
\end{array}\right] .
$$

The objective is to provide a control method to deal with systems subject to matched, $d_{2}$, and unmatched, $d_{1}$, timevarying bounded disturbances. We first discuss the case of matched disturbance and then we give a general method to deal with both types of disturbances.

1) ISS for time-varying matched disturbance: Interestingly, the system (31) subject to matched disturbance $d_{2}\left(d_{1}=0\right)$ is naturally ISS using the IC proposed in Proposition 3.2 with the $\mathrm{PCH}$ form (18) rewritten (to include the disturbance) as:

$\left[\begin{array}{c}\dot{x}_{q} \\ \dot{x}_{p} \\ \dot{x}_{v}\end{array}\right]=\left[\begin{array}{ccc}0 & M^{-1} M_{d} & -G K_{i} \\ -M_{d} M^{-1} & -R_{d} & 0 \\ K_{i} G^{\top} & 0 & 0\end{array}\right]\left[\begin{array}{c}\nabla_{x_{q}} H_{x} \\ \nabla_{x_{p}} H_{x} \\ \nabla_{x_{v}} H_{x}\end{array}\right]+\left[\begin{array}{c}0 \\ d_{2} \\ 0\end{array}\right]$.

This can be proven by taking

$$
H_{x}=\frac{1}{2} x_{p}^{\top} M_{d}^{-1} x_{p}+\frac{1}{2} x_{v}^{\top} K_{i}^{-1} x_{v}+V\left(x_{q}\right)
$$

as the candidate of the ISS-Lyapunov function. The Lyapunov derivative along the trajectories of (32) is computed as

$$
\begin{aligned}
\dot{H}_{x} & =x_{p}^{\top} M_{d}^{-1} \dot{x}_{p}+x_{v}^{\top} K_{i}^{-1} \dot{x}_{v}+\nabla V^{\top}\left(x_{q}\right) \dot{x}_{q} \\
& =-x_{p}^{\top} M_{d}^{-1} R_{d} M_{d}^{-1} x_{p}+x_{p}^{\top} M_{d}^{-1} d_{2} \\
& \leq-\lambda_{\min }\left(R_{d}\right)\left|M_{d}^{-1} x_{p}\right|^{2}+x_{p}^{\top} M_{d}^{-1} d_{2}
\end{aligned}
$$

where $\lambda_{\min }\left(R_{d}\right)$ is the smallest non-zero eigenvalue of $R_{d}$. Using the Young's inequality [7], rewritten as $-\zeta|y|^{2}+$ $\eta|y||z| \leq-\frac{\zeta}{2}|y|^{2}+\frac{\eta^{2}}{2 \zeta}|z|^{2}$, this yields

$$
\begin{aligned}
\dot{H}_{x} & \leq-\frac{\lambda_{\min }\left(R_{d}\right)}{2}\left|M_{d}^{-1} x_{p}\right|^{2}+\frac{1}{2 \lambda_{\min }\left(R_{d}\right)}\left|d_{2}\right|^{2} \\
& \leq-\alpha\left(\left|x_{p}\right|\right)+\sigma\left(\left|d_{2}\right|\right),
\end{aligned}
$$

with $\alpha, \sigma \in \mathcal{K}_{\infty}$. By invoking LaSalle's invariance principle, one can prove that the closed-loop PCH system is ISS w.r.t the matched disturbance (see the proof of Proposition 3.5).
2) ISS for time-varying matched and unmatched disturbances: Here we show the general case when both matched and unmatched disturbances are present. A method to deal with this situation has been recently reported in [7]. Unfortunately, this method results in a complicated closed-loop system, due to the complex augmented PCH structure and the complex controller. Furthermore, asymptotic stability has not been achieved unless $d_{1}=0$ and $d_{2}$ is constant i.e. only matched and time invariant disturbances. The following proposition shows our new approach, providing simpler ISS control design method as well as achieving asymptotic stability even in the presence of time-varying matched and unmatched disturbances.

Proposition 3.5: Consider the separable PCH system (31) with time-varying bounded disturbances $d_{1}, d_{2}$ and the control law

$$
\begin{aligned}
G u & =\left(\nabla_{q} H-M_{d} M^{-1} \nabla_{x_{q}} H_{x}-R_{d} M_{d}^{-1} p\right. \\
& \left.-R_{d} M_{d}^{-1} M G x_{v}-\rho\left|\nabla_{x_{q}} V\left(x_{q}\right)\right|^{2} \nabla_{x_{p}} H_{x}-M G \dot{x}_{v}\right) \\
\dot{x}_{v} & =K_{i} G^{T} \nabla_{x_{q}} H_{x} .
\end{aligned}
$$

There exist a constant $\rho>0$, such that using the state transformation (13) to realize the augmented closed-loop PCH system

$$
\begin{aligned}
& {\left[\begin{array}{c}
\dot{x}_{q} \\
\dot{x}_{p} \\
\dot{x}_{v}
\end{array}\right]=\left[\begin{array}{ccc}
0 & M^{-1} M_{d} & -G K_{i} \\
-M_{d} M^{-1} & -R_{d}-\rho\left|\nabla_{x_{q}} V\left(x_{q}\right)\right|^{2} & 0 \\
K_{i} G^{\top} & 0
\end{array}\right] } \\
& \times\left[\begin{array}{l}
\nabla_{x_{q}} H_{x} \\
\nabla_{x_{p}} H_{x} \\
\nabla_{x_{v}} H_{x}
\end{array}\right]+\left[\begin{array}{c}
d_{1} \\
d_{2} \\
0
\end{array}\right],
\end{aligned}
$$

with the desired Hamiltonian function (33), the perturbed closed-loop system (36) is ISS with respect to the disturbances $d_{1}$ and $d_{2}$. Moreover, the function (33) is the ISS-Lyapunov function for the system (36).

Proof of Proposition 3.5: Consider the desired Hamiltonian function (33) as a candidate ISS-Lyapunov function. Its timederivative along the trajectories of (36) along with (13) is given by

$$
\begin{aligned}
\dot{H}_{x}= & x_{p}^{\top} M_{d}^{-1} \dot{x}_{p}+x_{v}^{\top} K_{i}^{-1} \dot{x}_{v}+\nabla_{x_{q}} V\left(x_{q}\right)^{\top} \dot{x}_{q} \\
= & -x_{p}^{\top} M_{d}^{-1} R_{d} M_{d}^{-1} x_{p}+x_{p}^{\top} M_{d}^{-1} d_{2} \\
& -x_{p}^{\top} M_{d}^{-1} \rho\left|\nabla_{x_{q}} V\right|^{2} M_{d}^{-1} x_{p}+\nabla_{x_{q}} V^{\top} d_{1} \\
\leq & -\lambda_{\min }\left(R_{d}\right)\left|M_{d}^{-1} x_{p}\right|^{2}+x_{p}^{\top} M_{d}^{-1} d_{2} \\
& -\rho\left|x_{p}^{\top} M_{d}^{-1} \nabla_{x_{q}} V^{\top}\right|^{2}+\nabla_{x_{q}} V^{\top} d_{1} .
\end{aligned}
$$

Applying the Young's inequality, where the first two terms in the inequality (37) are treated as in (34), and using

$$
\nabla_{x_{q}} V^{\top} d_{1} \leq \frac{1}{2}\left|\nabla_{x_{q}} V^{\top}\right|^{2}+\frac{1}{2}\left|d_{1}\right|^{2},
$$

gives

$$
\begin{aligned}
\dot{H}_{x} \leq & -\frac{\lambda_{\min }\left(R_{d}\right)}{2}\left|M_{d}^{-1} x_{p}\right|^{2}+\frac{1}{2 \lambda_{\min }\left(R_{d}\right)}\left|d_{2}\right|^{2} \\
& -\frac{1}{2} \rho\left|x_{p}^{\top} M_{d}^{-1} \nabla_{x_{q}} V^{\top}\right|^{2}+\frac{1}{2}\left|d_{1}\right|^{2} \\
& -\frac{1}{2} \rho\left|x_{p}^{\top} M_{d}^{-1} \nabla_{x_{q}} V^{\top}\right|^{2}+\frac{1}{2}\left|\nabla_{x_{q}} V^{\top}\right|^{2} .
\end{aligned}
$$


Choosing a sufficiently large $\rho$, the term $\frac{1}{2} \rho\left|x_{p}^{\top} M_{d}^{-1} \nabla_{x_{q}} V^{\top}\right|^{2}$ dominates $\frac{1}{2}\left|\nabla_{x_{q}} V^{\top}\right|^{2}$. Thus, we have

$$
\dot{H}_{x} \leq-\alpha\left(\left|x_{q}, x_{p}\right|\right)+\sigma(|d|),
$$

with $\alpha, \sigma \in \mathcal{K}_{\infty}$. As (39) is only negative semidefinite w.r.t. $x$, we invoke LaSalle's invariance principle to complete the proof. For $d=0, \dot{H}_{x}=0$ implies that

$$
\begin{aligned}
-\frac{\lambda_{\min }\left(R_{d}\right)}{2}\left|M_{d}^{-1} x_{p}\right|^{2}-\frac{1}{2} \rho\left|x_{p}^{\top} M_{d}^{-1} \nabla_{x_{q}} V^{\top}\right|^{2} & =0 \\
-\frac{1}{2}\left|M_{d}^{-1} x_{p}\right|^{2}\left(\lambda_{\min }\left(R_{d}\right)+\rho\left|\nabla_{x_{q}} V^{\top}\right|^{2}\right) & =0 .
\end{aligned}
$$

Because the terms inside the brackets are always positive, the only possible solutions for (40) is $\left|M_{d}^{-1} x_{p}\right|^{2}=0$. This implies $x_{p} \equiv 0 \Longrightarrow \dot{x}_{p} \equiv 0 \Longrightarrow \nabla_{x_{q}} H_{x}=0$.

Invoking similar arguments to those in the proof of Proposition 3.2, we obtain

$$
\nabla_{x_{q}} H_{x} \equiv 0 \Longrightarrow x_{q}=q_{e} \text { and } x_{v}=0 .
$$

Now, from (38) and the fact the $H_{x}$ function is positive definite, proper and has an isolated minimum (5) as a consequence of using IDA-PBC method, all conditions of the ISS property (see [18, Section 3.3] and [19, Remark 2.4]) are satisfied, which proves that the closed-loop PCH system is ISS w.r.t the matched and unmatched disturbances.

Remark 3.6: Proposition 3.5 can be extended to apply to underactuated $\mathrm{PCH}$ mechanical systems by replacing the matrix $G$ in (36) with $\mathcal{G}$ as in (24), in a similar way as the results in Section $\amalg I-C$

\section{E. Adaptive IDA-PBC control for separable PCH systems}

The implementation of the IDA-PBC controller (6) requires the exact knowledge of the system's parameters, essentially the inertia matrix $M$ and the potential energy function $V$. Neglecting parameter uncertainties may deteriorate the stability and/or the transient behavior of the control system. This motivates the establishment of an adaptive scheme to estimate the uncertainties.

A common occurrence of uncertainties in PCH models is the uncertainty in the potential energy function, thus in the gradient of this function. The linearly parameterized gradient of the potential energy function can then be written as

$$
\nabla_{q} V(q)=F(q) \theta,
$$

where the matrix function $F(q)$ is known and the vector $\theta$ contains the unknown, but constant parameters. Notice that in the IDA-PBC method the desired potential energy function $V_{d}(q)$ (the second term in (4)) contains $V(q)$ in its terms. Therefore, the gradient of this function can be written as

$$
\nabla_{q} V_{d}(q)=\nabla_{q} V(q)+S(q)=F(q) \theta+S(q),
$$

where $S(q)$ is known. To deal with the class of PCH systems with this type of uncertainty, we propose two adaptive-IDAPBC control methods. In the first method, the integral action is applied on the passive outputs, while in the second method is applied on the non-passive outputs and includes a change of coordinates.
1) Integral control on passive outputs:

Proposition 3.6: Consider the separable PCH system (1). The potential energy function $V(q)$ of the system contains uncertainties, hence its gradient can be represented as in (41). Define the augmented closed-loop PCH system as

$$
\left[\begin{array}{c}
\dot{q} \\
\dot{p} \\
\dot{\hat{\theta}}
\end{array}\right]=\left[\begin{array}{ccc}
0 & M^{-1} M_{d} & 0 \\
-M_{d} M^{-1} & -R_{d} & Q F(q) \\
0 & -(Q F(q))^{\top} & 0
\end{array}\right]\left[\begin{array}{c}
\nabla_{q} H_{d} \\
\nabla_{p} H_{d} \\
\nabla_{\tilde{\theta}} H_{d}
\end{array}\right],
$$

where $\hat{\theta}$ is the estimate of $\theta, \tilde{\theta}=\hat{\theta}-\theta$ is the estimation error, $Q=\left(I_{n}-M_{d} M^{-1}\right)$ and

$$
H_{d}=\frac{1}{2} p^{\top} M_{d}^{-1} p+\frac{1}{2}|\tilde{\theta}|^{2}+V_{d}(q)
$$

is the desired Hamiltonian function. Then, the controller

$$
G u=F \hat{\theta}-M_{d} M^{-1} F \hat{\theta}-M_{d} M^{-1} S-R_{d} M_{d}^{-1} p,
$$

with the update law

$$
\dot{\hat{\theta}}=-(Q F)^{\top} \nabla_{p} H_{d},
$$

stabilizes the system at the equilibrium $\left(q_{e}, 0, \theta\right)$.

Proof of Proposition 3.6: Consider the desired Hamiltonian function (44) as a candidate Lyapunov function. Its time derivative along the trajectories of (43) satisfies

$$
\begin{aligned}
\dot{H}_{d} & =p^{\top} M_{d}^{-1} \dot{p}+\tilde{\theta}^{\top} \dot{\tilde{\theta}}+\nabla_{q} V_{d}^{\top} \dot{q} \\
= & p^{\top} M_{d}^{-1}\left(-M_{d} M^{-1} \nabla_{q} V_{d}-R_{d} \nabla_{p} H_{d}+Q F \nabla_{\tilde{\theta}} H_{d}\right) \\
& -\tilde{\theta}^{\top}\left(F^{\top} Q^{\top} \nabla_{p} H_{d}\right)+\nabla_{q} V_{d}^{\top}\left(M^{-1} M_{d} \nabla_{p} H_{d}\right) \\
= & -p^{\top} M^{-1} \nabla_{q} V_{d}-p^{\top} M_{d}^{-1} R_{d} M_{d}^{-1} p+p^{\top} M_{d}^{-1} Q F \tilde{\theta} \\
& -\tilde{\theta}^{\top} F^{\top} Q^{\top} M_{d}^{-1} p+\nabla_{q} V_{d}^{\top} M^{-1} p \\
= & -p^{\top} M^{-1} \nabla_{q} V_{d}+\left(p^{\top} M^{-1} \nabla_{q} V_{d}\right)^{\top}+p^{\top} M_{d}^{-1} Q F \tilde{\theta} \\
& -\left(p^{\top} M_{d}^{-1} Q F \tilde{\theta}\right)^{\top}-p^{\top} M_{d}^{-1} R_{d} M_{d}^{-1} p \\
= & -p^{\top} M_{d}^{-1} R_{d} M_{d}^{-1} p \leq-\left|M_{d}^{-1} p\right|_{R_{d}}^{2} \leq 0 .
\end{aligned}
$$

Notice that from (47), we have $\dot{H}_{d}=0 \Longrightarrow p=0$. Furthermore, $p \equiv 0 \Longrightarrow \dot{p} \equiv 0$. Thus, under the dynamics (43) yields:

$$
\begin{aligned}
\dot{p}= & -M_{d} M^{-1} \nabla_{q} V_{d}-R_{d} \nabla_{p} H_{d}+Q F(q) \nabla_{\tilde{\theta}} H_{d} \\
= & -M_{d} M^{-1}(F \theta+S(q))-R_{d} M_{d}^{-1} p+Q F \tilde{\theta} \\
= & -M_{d} M^{-1}(F \theta+S)+\left(I_{n}-M_{d} M^{-1}\right) F(\hat{\theta}-\theta) \\
= & -M_{d} M^{-1} F \theta+M_{d} M^{-1} F \theta-M_{d} M^{-1} S-M_{d} M^{-1} F \hat{\theta} \\
& +F \hat{\theta}-F \theta=0 \\
= & -M_{d} M^{-1} S-M_{d} M^{-1} F \hat{\theta}+F \hat{\theta}-F \theta=0 .
\end{aligned}
$$

From (44) and (47), $p \in \mathcal{L}_{2} \cap \mathcal{L}_{\infty}$ and $q, \tilde{\theta} \in \mathcal{L}_{\infty}$. Therefore, the zero momentum (velocity) may guarantee bounded $\tilde{\theta}$ and consequently the convergence of the position states to their desired values but with steady-state errors. That is, the smallest $\tilde{\theta} \Longrightarrow \hat{\theta} \approx \theta$. Now, from (48) this implies that $F \hat{\theta}+S \approx 0$. Since $F \hat{\theta}+S=\nabla_{q} H_{d}=0$, using similar arguments as in the proof of Proposition 3.2, we have

$$
\nabla_{q} H_{d} \equiv 0 \Longrightarrow q=q_{e} .
$$

This shows that some asymptotic properties of the proposed design method can be concluded. 
2) Integral control on non-passive outputs: Another method to design an adaptive controller is by applying the integral action on the non-passive outputs. Thus, we obtain the following closed-loop PCH model:

$$
\left[\begin{array}{c}
\dot{q} \\
\dot{p} \\
\dot{\hat{\theta}}
\end{array}\right]=\left[\begin{array}{ccc}
0 & M^{-1} M_{d} & \mathcal{U} \\
-M_{d} M^{-1} & -R_{d} & 0 \\
-\mathcal{U} & 0 & 0
\end{array}\right]\left[\begin{array}{c}
\nabla_{q} H_{d} \\
\nabla_{p} H_{d} \\
\nabla_{\tilde{\theta}} H_{d}
\end{array}\right] .
$$

Where $\mathcal{U}$ is a constant matrix. Two problems arise from this method; 1) the update law $\dot{\hat{\theta}}=-\mathcal{U} \nabla_{q} H_{d}=-\mathcal{U}(F(q) \theta+$ $S(q)$ ), is a function of the unknown $\theta$, and 2) the integral action is unattainable from the closed-loop system. To solve these problems we propose a change of coordinates similar to those proposed in the previous sections, aiming at asymptotically stabilizing the uncertain system (1) at the the equilibrium point $\left(q_{e}, 0, \theta\right)$.

Proposition 3.7: Consider the separable PCH system (1). The potential energy function $V(q)$ of the system contains uncertainties, hence its gradient can be represented as in (41). Define the state transformation

$$
x_{q}=q ; \quad x_{p}=p-\mathcal{U} \tilde{\theta} ; \quad x_{\tilde{\theta}}=\tilde{\theta},
$$

to realize the augmented closed-loop PCH system

$$
\left[\begin{array}{c}
\dot{x}_{q} \\
\dot{x}_{p} \\
\dot{x}_{\hat{\theta}}
\end{array}\right]=\left[\begin{array}{ccc}
0 & M^{-1} M_{d} & M^{-1} \mathcal{U} \\
-M_{d} M^{-1} & -R_{d} & \Lambda \\
-M^{-1} \mathcal{U} & -\Lambda & -\Upsilon
\end{array}\right]\left[\begin{array}{c}
\nabla_{x_{q}} H_{x} \\
\nabla_{x_{p}} H_{x} \\
\nabla_{x_{\tilde{\theta}}} H_{x}
\end{array}\right] .
$$

where $\hat{\theta}$ is the estimate of $\theta, \tilde{\theta}=\hat{\theta}-\theta$ is the estimation error, $R_{d}, \Upsilon>0$,

$$
\begin{aligned}
& \Lambda=F(q)-M_{d} M^{-1} F(q)-R_{d} M_{d}^{-1} \mathcal{U} \\
& \Upsilon=M^{-1} \mathcal{U} F(q)+\Lambda M_{d}^{-1} \mathcal{U}
\end{aligned}
$$

and

$$
H_{x}=\frac{1}{2} x_{p}^{\top} M_{d}^{-1} x_{p}+\frac{1}{2}|\tilde{\theta}|^{2}+V\left(x_{q}\right),
$$

is the desired Hamiltonian function. Then, the controller

$G u=F(q) \hat{\theta}-M_{d} M^{-1} F(q) \hat{\theta}-M_{d} M^{-1} S(q)-R_{d} M_{d}^{-1} p+\mathcal{U} \dot{\hat{\theta}}$

with the update law

$$
\dot{x}_{\hat{\theta}}=\dot{\hat{\theta}}=-M^{-1} \mathcal{U} F(q) \hat{\theta}-M^{-1} \mathcal{U} S(q)-\Lambda M_{d}^{-1} p
$$

asymptotically stabilizes the system at the equilibrium $\left(q_{e}, 0, \theta\right)$.

Proof of Proposition 3.7; The proof is established by (i) verifying the coincidence of the position and momenta states of system (1) with their corresponding states in (51). (ii) Showing that the expression of the update law $\dot{x}_{\hat{\theta}}$ doesn't depend on $\theta$. (iii) Showing that the proposed method achieves asymptotic stability.

(i) For the position states $q$, we have

$$
\begin{aligned}
\dot{x}_{q} & =M^{-1} M_{d} \nabla_{x_{p}} H_{x}+M^{-1} \mathcal{U} \nabla_{x_{\tilde{\theta}}} H_{x} \\
& =M^{-1} M_{d} M_{d}^{-1} x_{p}+M^{-1} \mathcal{U} \tilde{\theta} \\
& =M^{-1}(p-\mathcal{U} \tilde{\theta})+M^{-1} \mathcal{U} \tilde{\theta}=M^{-1} p \equiv \dot{q},
\end{aligned}
$$

and for the momenta $p$,

$$
\begin{aligned}
\dot{p} \equiv & \dot{x}_{p}+\mathcal{U} \dot{\hat{\theta}} \quad(\dot{\tilde{\theta}}=\dot{\hat{\theta}} \text { as } \theta \text { is constant }) \\
G u-\nabla_{q} H= & -M_{d} M^{-1} \nabla_{x_{q}} H_{x}-R_{d} \nabla_{x_{p}} H_{x}+\Lambda \nabla_{x_{\tilde{\theta}}} H_{x} \\
& +\mathcal{U} \dot{\hat{\theta}} \\
G u-F \theta= & -M_{d} M^{-1}(F \theta+S)-R_{d} M_{d}^{-1}(p-\mathcal{U} \tilde{\theta}) \\
& +\left(F-M_{d} M^{-1} F-R_{d} M_{d}^{-1} \mathcal{U}\right) \tilde{\theta}+\mathcal{U} \dot{\hat{\theta}} .
\end{aligned}
$$

Using $(\tilde{\theta}=\hat{\theta}-\theta)$ and solving, we obtain (53).

(ii) The update law is given by

$$
\begin{aligned}
\dot{x}_{\hat{\theta}}= & -M^{-1} \mathcal{U} \nabla_{x_{q}} H_{x}-\Lambda \nabla_{x_{p}} H_{x}-\Upsilon \nabla_{x_{\tilde{\theta}}} H_{x} \\
= & -M^{-1} \mathcal{U}(F \theta+S)-\Lambda M_{d}^{-1}(p-\mathcal{U} \tilde{\theta}) \\
& -\left(M^{-1} \mathcal{U} F+\Lambda M_{d}^{-1} \mathcal{U}\right) \tilde{\theta} \\
= & -M^{-1} \mathcal{U}(F \theta+S)-\Lambda M_{d}^{-1} p+\Lambda M_{d}^{-1} \mathcal{U} \tilde{\theta}-M^{-1} \mathcal{U} F \tilde{\theta} \\
& -\Lambda M_{d}^{-1} \mathcal{U} \tilde{\theta} \\
= & -M^{-1} \mathcal{U} F \theta-M^{-1} \mathcal{U} S-\Lambda M_{d}^{-1} p-M^{-1} \mathcal{U} F \tilde{\theta} .
\end{aligned}
$$

Substituting $(\tilde{\theta}=\hat{\theta}-\theta)$ in the last row in (55), yields

$$
\begin{aligned}
\dot{x}_{\hat{\theta}}= & -M^{-1} \mathcal{U} F \theta-M^{-1} \mathcal{U} S-\Lambda M_{d}^{-1} p-M^{-1} \mathcal{U} F \hat{\theta} \\
& +M^{-1} \mathcal{U} F \theta .
\end{aligned}
$$

Canceling like terms but with opposite signs, we obtain (54).

(iii) Consider the function (52), whose time-derivative along the trajectories of (51) along with (50) is

$$
\begin{aligned}
\dot{H}_{x}= & \left(\nabla_{x_{p}} H_{x}\right)^{\top} \dot{x}_{p}+\left(\nabla_{x_{\tilde{\theta}}} H_{x}\right)^{\top} \dot{x}_{\hat{\theta}}+\left(\nabla_{x_{q}} H_{x}\right)^{\top} \dot{x}_{q} \\
= & \nabla_{x_{p}} H_{x}^{\top}\left(-M_{d} M^{-1} \nabla_{x_{q}} H_{x}-R_{d} \nabla_{x_{p}} H_{x}+\Lambda \nabla_{x_{\tilde{\theta}}} H_{x}\right) \\
& +\nabla_{x_{\tilde{\theta}}} H_{x}^{\top}\left(-M^{-1} \mathcal{U} \nabla_{x_{q}} H_{x}-\Lambda \nabla_{x_{p}} H_{x}-\Upsilon \nabla_{x_{\tilde{\theta}}} H_{x}\right) \\
& +\nabla_{x_{q}} H_{x}^{\top}\left(M^{-1} M_{d} \nabla_{x_{p}} H_{x}+M^{-1} \mathcal{U} \nabla_{x_{\tilde{\theta}}} H_{x}\right) \\
= & -\nabla_{x_{p}} H_{x}^{\top} M_{d} M^{-1} \nabla_{x_{q}} H_{x}-\nabla_{x_{p}} H_{x}^{\top} R_{d} \nabla_{x_{p}} H_{x} \\
& +\nabla_{x_{p}} H_{x}^{\top} \Lambda \nabla_{x_{\tilde{\theta}}} H_{x}-\nabla_{x_{\tilde{\theta}}} H_{x}^{\top} M^{-1} \mathcal{U} \nabla_{x_{q}} H_{x} \\
& -\nabla_{x_{\tilde{\theta}}} H_{x}^{\top} \Lambda \nabla_{x_{p}} H_{x}-\nabla_{x_{\tilde{\theta}}} H_{x}^{\top} \Upsilon \nabla_{x_{\tilde{\theta}}} H_{x} \\
& +\nabla_{x_{q}} H_{x}^{\top} M^{-1} M_{d} \nabla_{x_{p}} H_{x}+\nabla_{x_{q}} H_{x}^{\top} M^{-1} \mathcal{U} \nabla_{x_{\tilde{\theta}}} H_{x} .
\end{aligned}
$$

Taking the transpose of the terms $\nabla_{x_{q}} H_{x}^{\top} M^{-1} M_{d} \nabla_{x_{p}} H_{x}$, $\nabla_{x_{\tilde{\theta}}} H_{x}^{\top} \Lambda \nabla_{x_{p}} H_{x}$ and $\nabla_{x_{q}} H_{x}^{\top} M^{-1} \mathcal{U} \nabla_{x_{\tilde{\theta}}} H_{x}$ and rearranging, yields

$$
\begin{aligned}
\dot{H}_{x}= & -\nabla_{x_{p}} H_{x}^{\top} M_{d} M^{-1} \nabla_{x_{q}} H_{x}+\left(\nabla_{x_{p}} H_{x}^{\top} M_{d} M^{-1} \nabla_{x_{q}} H_{x}\right)^{\top} \\
& +\nabla_{x_{p}} H_{x}^{\top} \Lambda \nabla_{x_{\tilde{\theta}}} H_{x}-\left(\nabla_{x_{p}} H_{x}^{\top} \Lambda \nabla_{x_{\tilde{\theta}}} H_{x}\right)^{\top} \\
& -\nabla_{x_{\tilde{\theta}}} H_{x}^{\top} \Upsilon \nabla_{x_{\tilde{\theta}}} H_{x}-\nabla_{x_{\tilde{\theta}}} H_{x}^{\top} M^{-1} \mathcal{U} \nabla_{x_{q}} H_{x} \\
& -\nabla_{x_{p}} H_{x}^{\top} R_{d} \nabla_{x_{p}} H_{x}+\left(-\nabla_{x_{\tilde{\theta}}} H_{x}^{\top} M^{-1} \mathcal{U} \nabla_{x_{q}} H_{x}\right)^{\top} \\
= & -\nabla_{x_{p}} H_{x}^{\top} R_{d} \nabla_{x_{p}} H_{x}-\nabla_{x_{\tilde{\theta}}} H_{x}^{\top} \Upsilon \nabla_{x_{\tilde{\theta}}} H_{x} \\
\leq & -\left|\nabla_{x_{p}} H_{x}\right|_{R_{d}}^{2}-\left|\nabla_{x_{\tilde{\theta}}} H_{x}\right|_{\Upsilon}^{2} .
\end{aligned}
$$

Thus, the system (51) has a stable equilibrium at $\left(q_{e}, 0, \theta\right)$ with $x_{p}, x_{\tilde{\theta}} \in \mathcal{L}_{2} \cap \mathcal{L}_{\infty}$. The convergence of the states is established invoking the following practical corollary of Barbalat's lemma [20]:

Corollary 3.1: Consider a function $\phi: \mathbb{R}_{>0} \rightarrow \mathbb{R}$ and suppose that $\phi(t) \in \mathcal{L}_{2} \cap \mathcal{L}_{\infty}$ and $\dot{\phi}(t) \in \mathcal{L}_{\infty}$. Then $\lim _{t \rightarrow \infty} \phi(t)=0$. 
Applying Corollary 3.1, implies the convergence of states $x_{p}$ and $x_{\tilde{\theta}}$ to zero $\left(x_{p}, x_{\tilde{\theta}} \rightarrow 0\right.$ as $\left.t \rightarrow \infty\right)$. Moreover, $\tilde{\theta}=0 \Longrightarrow$ $\hat{\theta}=\theta$. Finally, the convergence of the states $x_{q}$ to their desired states is established as follows:

$x_{p}, x_{\tilde{\theta}} \equiv 0 \Longrightarrow \dot{x}_{p}, \hat{\theta} \equiv 0$. Thus, under the dynamics of (51) yields:

$$
\begin{aligned}
\dot{x}_{p} & =-M_{d} M^{-1} \nabla_{x_{q}} H_{x}-R_{d} \nabla_{x_{p}} H_{x}+\Lambda \nabla_{x_{\tilde{\theta}}} H_{x}=0 \\
& =-M_{d} M^{-1} \nabla_{x_{q}} H_{x}-\underbrace{R_{d} M_{d}^{-1} x_{p}}_{=0}+\underbrace{\Lambda \tilde{\theta}}_{=0}=0 \\
& =-M_{d} M^{-1} \nabla_{x_{q}} H_{x}=0 .
\end{aligned}
$$

This implies $\nabla_{x_{q}} H_{x}=0$. Invoking similar arguments to those in the proof of Proposition 3.2, we obtain

$$
\nabla_{x_{q}} H_{x} \equiv 0 \Longrightarrow x_{q}=q_{e} .
$$

Since all trajectories converge to their desired values, we can conclude that the closed-loop system (51) has an asymptotically stable equilibrium at $\left(q_{e}, 0, \theta\right)$.

\section{Robust Control of Non-SEPARABLE HAMILTONIAN SYSTEMS}

In this section, we extend our results in Section [III to deal with non-separable $\mathrm{PCH}$ systems. In this case, as $M$ and $M_{d}$ are functions of $q$, their derivatives need to be taken into account in the construction of the control law. Fortunately, our approaches do not require significant changes in the interconnection matrices (thus the augmented closedloop PCH models) which have been constructed from their separable counterparts (in [7] significant changes applied). As expected, more complicated control laws are obtained as a consequence. Before we state the results, we show some necessary differentiations that are used throughout the proof of results. Similar to (13), we start with defining

$$
\begin{aligned}
x_{p} & =p+\mathcal{P} \\
\mathcal{P} & =M\left(x_{q}\right) G x_{v} .
\end{aligned}
$$

The derivative is obviously

$$
\dot{x}_{p}=\dot{p}+\dot{\mathcal{P}},
$$

while $\dot{x}_{p}$ and $\dot{p}$ are obtained directly from their corresponding PCH models. The term $\dot{\mathcal{P}}$ is calculated as

$$
\begin{aligned}
\dot{\mathcal{P}} & =\dot{M} G x_{v}+M G \dot{x}_{v}, \\
\dot{M} G x_{v} & =\sum_{i=1}^{n}\left(\nabla_{q_{i}} M G x_{v}\right)\left(e_{i}^{\top} \dot{x}_{q}\right) .
\end{aligned}
$$

Second, given non-constant $M\left(x_{q}\right)$ and $M_{d}\left(x_{q}\right)$, the derivative of the Hamiltonian function

$$
H_{x}=\frac{1}{2} x_{p}^{\top} M_{d}^{-1}\left(x_{q}\right) x_{p}+\frac{1}{2} x_{v}^{\top} K_{i}^{-1} x_{v}+V\left(x_{q}\right),
$$

becomes

$$
\begin{aligned}
\dot{H}_{x}= & \left(\nabla_{x_{p}} H_{x}\right)^{\top} \dot{x}_{p}+\left(\nabla_{x_{v}} H_{x}\right)^{\top} \dot{x}_{v}+\left(\nabla_{x_{q}} H_{x}\right)^{\top} \dot{x}_{q} \\
= & x_{p}^{\top} M_{d}^{-1} \dot{x}_{p}+x_{v}^{\top} K_{i}^{-1} \dot{x}_{v} \\
& +\left(\nabla_{x_{q}} V_{x}^{\top}+\frac{1}{2} \sum_{i=1}^{n} e_{i} x_{p}^{\top} \nabla_{q_{i}} M_{d}^{-1} x_{p}\right) \dot{x}_{q},
\end{aligned}
$$

where the derivative of $M_{d}$ is now taken into account.

\section{A. Integral IDA-PBC for non-separable PCH systems}

Proposition 4.1: Consider the non-separable PCH system (11). Assume a stabilizing IDA-PBC controller (6) has already been obtained with the desired (closed-loop) energy function (41) and the desired PCH dynamics take the form

$$
\left[\begin{array}{c}
\dot{q} \\
\dot{p}
\end{array}\right]=\left[\begin{array}{cc}
0 & M^{-1} M_{d}(q) \\
-M_{d} M^{-1}(q) & J_{2}-R_{d}
\end{array}\right]\left[\begin{array}{c}
\nabla_{q} H_{d} \\
\nabla_{p} H_{d}
\end{array}\right]+\left[\begin{array}{l}
0 \\
G
\end{array}\right] v .
$$

Defining the state transformation (13) to realize the augmented closed-loop PCH system

$$
\left[\begin{array}{c}
\dot{x}_{q} \\
\dot{x}_{p} \\
\dot{x}_{v}
\end{array}\right]=\left[\begin{array}{ccc}
0 & M^{-1} M_{d}\left(x_{q}\right) & -G K_{i} \\
-M_{d} M^{-1}\left(x_{q}\right) & J_{2}-R_{d} & 0 \\
K_{i} G^{\top} & 0 & 0
\end{array}\right]\left[\begin{array}{c}
\nabla_{x_{q}} H_{x} \\
\nabla_{x_{p}} H_{x} \\
\nabla_{x_{v}} H_{x}
\end{array}\right]_{(64)}
$$

with the Hamiltonian function (61), asymptotic stability of the equilibrium point $x_{e}=\left(q_{e}, 0,0\right)$ is preserved with the IC

$$
\begin{aligned}
G v= & -\frac{1}{2} M_{d} M^{-1} \sum_{i=1}^{n} e_{i} p^{\top} \nabla_{q_{i}} M_{d}^{-1} M G x_{v} \\
& -\frac{1}{2} M_{d} M^{-1} \sum_{i=1}^{n} e_{i} x_{v}^{\top} G M \nabla_{q_{i}} M_{d}^{-1} p \\
& -\frac{1}{2} M_{d} M^{-1} \sum_{i=1}^{n} e_{i} x_{v}^{\top} G M \nabla_{q_{i}} M_{d}^{-1} M G x_{v} \\
& -\sum_{i=1}^{n}\left(\frac{\partial M}{\partial x_{q_{i}}} G x_{v}\right)\left(e_{i}^{\top} \dot{x}_{q}\right) \\
& +\left(J_{2}-R_{d}\right) M_{d}^{-1} M G x_{v}-M G \dot{x}_{v}
\end{aligned}
$$

and $\quad \dot{x}_{v}=K_{i} G^{\top} \nabla_{x_{q}} H_{x}$.

Proof of Proposition 4.1; The proof can be established following the same procedures as in the proof of Proposition 3.2 The time derivative of the Hamiltonian function (61) along the trajectories of the system is

$$
\begin{aligned}
\dot{H}_{x}= & \nabla_{x_{p}} H_{x}^{\top} \dot{x}_{p}+\nabla_{x_{v}} H_{x}^{\top} \dot{x}_{v}+\nabla_{x_{q}} H_{x}^{\top} \dot{x}_{q} \\
= & -\nabla_{x_{p}} H_{x}^{\top} M_{d} M^{-1} \nabla_{x_{q}} H_{x}+\nabla_{x_{p}} H_{x}^{\top}\left(J_{2}-R_{d}\right) \nabla_{x_{p}} H_{x} \\
& +\nabla_{x_{v}} H_{x}^{\top} K_{i} G^{\top} \nabla_{x_{q}} H_{x}+\nabla_{x_{q}} H_{x}^{\top} M^{-1} M_{d} \nabla_{x_{p}} H_{x} \\
& -\nabla_{x_{q}} H_{x}^{\top} G K_{i} \nabla_{x_{v}} H_{x} .
\end{aligned}
$$

Taking a transpose of certain terms (to cancel out similar terms with different signs) and rearranging:

$$
\begin{aligned}
\dot{H}_{x}= & -\nabla_{x_{p}} H_{x}^{\top} M_{d} M^{-1} \nabla_{x_{q}} H_{x}+\left(\nabla_{x_{p}} H_{x}^{\top} M_{d} M^{-1} \nabla_{x_{q}} H_{x}\right)^{\top} \\
& +\nabla_{x_{v}} H_{x}^{\top} K_{i} G^{\top} \nabla_{x_{q}} H_{x}-\left(\nabla_{x_{v}} H_{x}^{\top} K_{i} G^{\top} \nabla_{x_{q}} H_{x}\right)^{\top} \\
& +\nabla_{x_{p}} H_{x}^{\top} J_{2} \nabla_{x_{p}} H_{x}-\nabla_{x_{p}} H_{x}^{\top} R_{d} \nabla_{x_{p}} H_{x} \\
= & \nabla_{x_{p}} H_{x}^{\top} J_{2} \nabla_{x_{p}} H_{x}-\nabla_{x_{p}} H_{x}^{\top} R_{d} \nabla_{x_{p}} H_{x} \\
= & -x_{p}^{\top} M_{d}^{-1} R_{d} M_{d}^{-1} x_{p} \leq 0 .
\end{aligned}
$$

Notice that because $J_{2}=-J_{2}^{\top}$, the term $\left(\nabla_{x_{p}} H_{x}\right)^{\top} J_{2} \nabla_{x_{p}} H_{x}$ is equal to zero. Furthermore, asymptotic stability is concluded by applying LaSalle's invariance principle:

$$
\begin{gathered}
-x_{p}^{\top} M_{d}^{-1} R_{d} M_{d}^{-1} x_{p}=0 \Longrightarrow x_{p}=\dot{x}_{p} \equiv 0 \\
\Longrightarrow M_{d}\left(x_{q}\right) M^{-1}\left(x_{q}\right) \nabla_{x_{q}} H_{x}=0 .
\end{gathered}
$$


Given $M\left(x_{q}\right), M_{d}\left(x_{q}\right)>0$, then we have $\nabla_{x_{q}} H_{x}=0$ which is only true if the system converges to its equilibrium point $q_{e}$. Thus,

$$
\nabla_{x_{q}} H_{x} \equiv 0 \Longrightarrow x_{q}=q_{e} \text { and } x_{v}=0,
$$

which proves that this equilibrium is asymptotically stable. The controller is obtained by matching the momenta of 630 and (64) along with the change of coordinates (13) and its time derivative (59), we get

$$
\begin{aligned}
\dot{p} & =-M_{d} M^{-1} \nabla_{q} H_{d}+J_{2} \nabla_{p} H_{d}-R_{d} \nabla_{p} H_{d}+G v \\
& \equiv \dot{x}_{p}-\dot{\mathcal{P}} \\
& =-M_{d} M^{-1} \nabla_{x_{q}} H_{x}+\left(J_{2}-R_{d}\right) \nabla_{x_{p}} H_{x}-\dot{\mathcal{P}} .
\end{aligned}
$$

Rearranging the terms,

$$
\begin{aligned}
G v & -\frac{1}{2} M_{d} M^{-1} \sum_{i=1}^{n} e_{i} p^{\top} \nabla_{q_{i}} M_{d}^{-1} p-M_{d} M^{-1} \nabla_{q} V_{d} \\
& +\left(J_{2}-R_{d}\right) M_{d}^{-1} p \equiv-\frac{1}{2} M_{d} M^{-1} \sum_{i=1}^{n} e_{i} x_{p}^{\top} \nabla_{q_{i}} M_{d}^{-1} x_{p} \\
& -M_{d} M^{-1} \nabla_{q} V_{d}+\left(J_{2}-R_{d}\right) M_{d}^{-1} x_{p}-\dot{\mathcal{P}} .
\end{aligned}
$$

Now, Substituting (58) and (60) in (67) and computing we obtain

$$
\begin{aligned}
G v & -\frac{1}{2} M_{d} M^{-1} \sum_{i=1}^{n} e_{i} p^{\top} \nabla_{q_{i}} M_{d}^{-1} p-M_{d} M^{-1} \nabla_{q} V_{d} \\
+ & \left(J_{2}-R_{d}\right) M_{d}^{-1} p \equiv-\frac{1}{2} M_{d} M^{-1}\left(p^{\top} \nabla_{q} M_{d}^{-1} p\right) \\
& -\frac{1}{2} M_{d} M^{-1} \sum_{i=1}^{n} e_{i} p^{\top}\left(\nabla_{q_{i}} M_{d}^{-1}\right) M G x_{v} \\
& -\frac{1}{2} M_{d} M^{-1} \sum_{i=1}^{n} e_{i} x_{v}^{\top} G M\left(\nabla_{q_{i}} M_{d}^{-1}\right) p \\
& -\frac{1}{2} M_{d} M^{-1} \sum_{i=1}^{n} e_{i} x_{v}^{\top} G M\left(\nabla_{q_{i}} M_{d}^{-1}\right) M G x_{v} \\
& -M_{d} M^{-1} \nabla_{q} V_{d}+\left(J_{2}-R_{d}\right)\left(M_{d}^{-1} p+M_{d}^{-1} M G x_{v}\right) \\
& -\sum_{i=1}^{n}\left(\frac{\partial M}{\partial x_{q_{i}}} G x_{v}\right)\left(e_{i}^{\top} \dot{x}_{q}\right)-M G \dot{x}_{v} .
\end{aligned}
$$

Canceling the similar terms on the left-hand side and righthand side of (68), we get the control law (65) and thus the proof is completed.

\section{B. ISS for non-separable PCH systems using IDA-PBC method}

The case of time-varying matched disturbance is treated following the same procedures of its separable counterparts, and taking into account the derivatives of $M$ and $M_{d}$. Here, we present the general case of time-varying matched and unmatched disturbances.

Proposition 4.2: Consider the non-separable PCH system (31) with time-varying bounded disturbances $d_{1}, d_{2}$ and the control law

$$
\begin{aligned}
G u= & \nabla_{q} H-M_{d} M^{-1} \nabla_{x_{q}} H_{x}+\left(J_{2}-R_{d}\right) M_{d}^{-1} p \\
& +\left(J_{2}-R_{d}\right) M_{d}^{-1} M G x_{v}+\rho T^{2} \nabla_{x_{p}} H_{x} \\
& -\sum_{i=1}^{n}\left(\nabla_{q_{i}} M G x_{v}\right)\left(e_{i}^{\top} M^{-1} M_{d} \nabla_{x_{p}} H_{x}\right) \\
& +\sum_{i=1}^{n}\left(\nabla_{q_{i}} M G x_{v}\right)\left(e_{i}^{\top} G K_{i} \nabla_{x_{v}} H_{x}\right)-M G \dot{x}_{v} \\
\dot{x}_{v}= & K_{i} G^{T} \nabla_{x_{q}} H_{x},
\end{aligned}
$$

with $T=\left|\nabla_{x_{p}} H_{x}^{\top} \sum_{i=1}^{n} \nabla_{q} M G x_{v}+\nabla_{x_{q}} H_{x}^{\top}\right|$. There exist a constant $\rho>0$, such that using the state transformation (13) to realize the augmented closed-loop PCH system

$$
\begin{aligned}
{\left[\begin{array}{c}
\dot{x}_{q} \\
\dot{x}_{p} \\
\dot{x}_{v}
\end{array}\right]=} & {\left[\begin{array}{ccc}
0 & M^{-1} M_{d} & -G K_{i} \\
-M_{d} M^{-1} & J_{2}-R_{d}+\rho T^{2} & 0 \\
K_{i} G^{\top} & 0 & 0
\end{array}\right]\left[\begin{array}{c}
\nabla_{x_{q}} H_{x} \\
\nabla_{x_{p}} H_{x} \\
\nabla_{x_{v}} H_{x}
\end{array}\right] } \\
& +\left[\begin{array}{cc}
d_{1} \\
d_{2}+\sum_{i=1}^{n} \nabla_{q_{i}} M G x_{v}\left(e_{i}^{\top} d_{1}\right) \\
0
\end{array}\right]
\end{aligned}
$$

and the desired Hamiltonian function 61), the perturbed closed-loop system (70) is ISS with respect to the disturbances $d_{1}$ and $d_{2}$. Moreover, the function (61) is the ISS-Lyapunov function for the system (70).

Proof of Proposition 4.2; Consider the desired Hamiltonian function (61) as a candidate ISS-Lyapunov function. Its timederivative along the trajectories of (70) along with (13) is given by

$$
\begin{aligned}
\dot{H}_{x}= & \left(\nabla_{x_{p}} H_{x}\right)^{\top} \dot{x}_{p}+\left(\nabla_{x_{v}} H_{x}\right)^{\top} \dot{x}_{v}+\left(\nabla_{x_{q}} H_{x}\right)^{\top} \dot{x}_{q} \\
= & -\nabla_{x_{p}} H_{x}^{\top} M_{d} M^{-1} \nabla_{x_{q}} H_{x}+\nabla_{x_{p}} H_{x}^{\top}\left(J_{2}-R_{d}\right) \nabla_{x_{p}} H_{x} \\
& +\nabla_{x_{p}} H_{x}^{\top} \rho T \nabla_{x_{p}} H_{x}+\nabla_{x_{p}} H_{x}^{\top} d_{2} \\
& +\nabla_{x_{p}} H_{x}^{\top} \sum_{i=1}^{n}\left(\nabla_{q_{i}} M G x_{v}\right)\left(e_{i}^{\top} d_{1}\right) \\
& +\nabla_{x_{v}} H_{x}^{\top} K_{i} G^{\top} \nabla_{x_{q}} H_{x}+\nabla_{x_{q}} H_{x}^{\top} M^{-1} M_{d} \nabla_{x_{p}} H_{x} \\
& -\nabla_{x_{q}} H_{x}^{\top} G K_{i} \nabla_{x_{v}} H_{x}+\nabla_{x_{q}} H_{x}^{\top} d_{1} \\
= & -x_{p}^{\top} M_{d}^{-1} R_{d} M_{d}^{-1} x_{p}+x_{p}^{\top} M_{d}^{-1} d_{2} \\
& -x_{p}^{\top} M_{d}^{-1} \rho T^{2} M_{d}^{-1} x_{p}+T d_{1} \\
\leq & -\lambda_{\min }\left(R_{d}\right)\left|M_{d}^{-1} x_{p}\right|^{2}+x_{p}^{\top} M_{d}^{-1} d_{2} \\
& -\rho\left|x_{p}^{\top} M_{d}^{-1} T\right|^{2}+T d_{1} .
\end{aligned}
$$

Applying the Young's inequality, where the first two terms in the inequality (71) are treated as in 34, and using

$$
T d_{1} \leq \frac{1}{2}|T|^{2}+\frac{1}{2}\left|d_{1}\right|^{2},
$$

gives

$$
\begin{aligned}
\dot{H}_{x} \leq & -\frac{\lambda_{\min }\left(R_{d}\right)}{2}\left|M_{d}^{-1} x_{p}\right|^{2}+\frac{1}{2 \lambda_{\min }\left(R_{d}\right)}\left|d_{2}\right|^{2} \\
& -\frac{1}{2} \rho\left|x_{p}^{\top} M_{d}^{-1} T\right|^{2}+\frac{1}{2}\left|d_{1}\right|^{2} \\
& -\frac{1}{2} \rho\left|x_{p}^{\top} M_{d}^{-1} T\right|^{2}+\frac{1}{2}|T|^{2} .
\end{aligned}
$$


Choosing a sufficiently large $\rho$, the term $\frac{1}{2} \rho\left|x_{p}^{\top} M_{d}^{-1} T\right|^{2}$ dominates $\frac{1}{2}|T|^{2}$. Thus, we have

$$
\dot{H}_{x} \leq-\alpha\left(\left|x_{q}, x_{p}\right|\right)+\sigma(|d|),
$$

with $\alpha, \sigma \in \mathcal{K}_{\infty}$. As 73 is only negative semidefinite with respect to. $x$, we invoke LaSalle's invariance principle to complete the proof. For $d=0, \dot{H}_{x}=0$ implies that

$$
\begin{aligned}
-\frac{\lambda_{\min }\left(R_{d}\right)}{2}\left|M_{d}^{-1} x_{p}\right|^{2}-\frac{1}{2} \rho\left|x_{p}^{\top} M_{d}^{-1} T\right|^{2} & =0 \\
-\frac{1}{2}\left|M_{d}^{-1} x_{p}\right|^{2}\left(\lambda_{\min }\left(R_{d}\right)+\rho|T|^{2}\right) & =0 .
\end{aligned}
$$

Because the terms inside the brackets are always positive, the only possible solution for (74) is $\left|M_{d}^{-1} x_{p}\right|^{2}=0$. This implies $x_{p} \equiv 0 \Longrightarrow \dot{x}_{p} \equiv 0 \Longrightarrow \nabla_{x_{q}} H_{x}=0$. Invoking similar arguments to those in the proof of Proposition 3.2, we obtain

$$
\nabla_{x_{q}} H_{x} \equiv 0 \Longrightarrow x_{q}=q_{e} \text { and } x_{v}=0 .
$$

Now, from (72) and the fact the $H_{x}$ function is positive definite, proper and has an isolated minimum (5) as a consequence of using IDA-PBC method, all conditions of the ISS property (see [18, Section 3.3] and [19, Remark 2.4]) are satisfied, which proves that the closed-loop PCH system is ISS with respect to the matched and unmatched disturbances.

\section{Adaptive IDA-PBC control for non-separable PCH systems}

The case of integral control on passive outputs is treated following the same procedures of its separable counterparts, and taking into account the derivatives of $M$ and $M_{d}$. Here, we present the case of non-passive outputs, which is usually the case of interest.

Proposition 4.3: Consider the nonseparable PCH system (1). The potential energy function $V(q)$ of the system contains uncertainties, hence its gradient can be represented as in (41). Define the state transformation

$$
x_{q}=q ; \quad x_{p}=p-\mathcal{U} \tilde{\theta} ; \quad x_{\tilde{\theta}}=\tilde{\theta},
$$

to realize the augmented closed-loop PCH system

$$
\left[\begin{array}{c}
\dot{x}_{q} \\
\dot{x}_{p} \\
\dot{x}_{\hat{\theta}}
\end{array}\right]=\left[\begin{array}{ccc}
0 & M^{-1} M_{d} & M^{-1} \mathcal{U} \\
-M_{d} M^{-1} & -R_{d} & \Lambda \\
-M^{-1} \mathcal{U} & -\Lambda & -\Upsilon
\end{array}\right]\left[\begin{array}{c}
\nabla_{x_{q}} H_{x} \\
\nabla_{x_{p}} H_{x} \\
\nabla_{x_{\tilde{\theta}}} H_{x}
\end{array}\right],
$$

where $\hat{\theta}$ is the estimate of $\theta, \tilde{\theta}=\hat{\theta}-\theta$ is the estimation error, $R_{d}, \Upsilon>0$,

$$
\begin{aligned}
\Lambda= & F(q)-M_{d} M^{-1} F(q)+\left(J_{2}-R_{d}\right) M_{d}^{-1} \mathcal{U} \\
& -M_{d} M^{-1} \sum_{i=1}^{n} e_{i} p^{\top} \nabla_{q_{i}} M_{d}^{-1} \mathcal{U} \\
& +\frac{1}{2} M_{d} M^{-1} \sum_{i=1}^{n} e_{i}(\mathcal{U} \tilde{\theta})^{\top} \nabla_{q_{i}} M_{d}^{-1} \mathcal{U} \\
\Upsilon= & F(q) M_{d}^{-1} \mathcal{U}-\left(J_{2}-R_{d}\right)\left(M_{d}^{-1} \mathcal{U}\right)^{2} \\
& -\frac{1}{2} M^{-1} \mathcal{U} \sum_{i=1}^{n} e_{i} p^{\top} \nabla_{q_{i}} M_{d}^{-1} \mathcal{U}
\end{aligned}
$$

and

$$
H_{x}=\frac{1}{2} x_{p}^{\top} M_{d}^{-1} x_{p}+\frac{1}{2}|\tilde{\theta}|^{2}+V\left(x_{q}\right),
$$

is the desired Hamiltonian function. Then, the controller

$$
\begin{aligned}
G u= & F(q) \hat{\theta}-M_{d} M^{-1}(F(q) \hat{\theta}+S(q))+\left(J_{2}-R_{d}\right) M_{d}^{-1} p \\
& +\frac{1}{2} \sum_{i=1}^{n} e_{i} p^{\top} \nabla_{q_{i}} M^{-1} p \\
& -\frac{1}{2} M_{d} M^{-1} \sum_{i=1}^{n} e_{i} p^{\top} \nabla_{q_{i}} M_{d}^{-1} p+\mathcal{U} \dot{\hat{\theta}}
\end{aligned}
$$

with the update law

$$
\begin{aligned}
\dot{x}_{\hat{\theta}}= & -M^{-1} \mathcal{U}(F(q) \hat{\theta}+S(q))+\left(M^{-1}-M_{d}^{-1}\right) F(q) p \\
& +\frac{1}{2} M^{-1} \mathcal{U} \sum_{i=1}^{n} e_{i} p^{\top} \nabla_{q_{i}} M_{d}^{-1} p,
\end{aligned}
$$

asymptotically stabilizes the system at the equilibrium $\left(q_{e}, 0, \theta\right)$.

\section{Proof of Proposition 4.3.}

Following the same procedures as in the proof of Proposition 3.7, the time derivative of the Hamiltonian function (79) along the trajectories of the system is obtained as

$$
\begin{aligned}
\dot{H}_{x} & =-\nabla_{x_{p}} H_{x}^{\top}\left(J_{2}-R_{d}\right) \nabla_{x_{p}} H_{x}-\nabla_{x_{\tilde{\theta}}} H_{x}^{\top} \Upsilon \nabla_{x_{\tilde{\theta}}} H_{x} \\
& \leq-\left|\nabla_{x_{p}} H_{x}\right|_{R_{d}}^{2}-\left|\nabla_{x_{\tilde{\theta}}} H_{x}\right|_{\Upsilon}^{2},
\end{aligned}
$$

where the term $\left(\nabla_{x_{p}} H_{x}\right)^{\top} J_{2} \nabla_{x_{p}} H_{x}$ is equal to zero because $J_{2}=-J_{2}^{\top}$. Thus, the system (76) has a stable equilibrium at $\left(q_{e}, 0, \theta\right)$ with $x_{p}, x_{\tilde{\theta}} \in \mathcal{L}_{2} \cap \mathcal{L}_{\infty}$. By applying Corollary 3.1 we can conclude the convergence of states $x_{p}$ and $x_{\tilde{\theta}}$ to zero $\left(x_{p}, x_{\tilde{\theta}} \rightarrow 0\right.$ as $\left.t \rightarrow \infty\right)$. Moreover, $\tilde{\theta}=0 \Longrightarrow \hat{\theta}=\theta$. The convergence of the states $x_{q}$ to their desired equilibrium is established as follows:

$$
x_{p}, x_{\tilde{\theta}} \equiv 0 \Longrightarrow \dot{x}_{p}, \dot{\hat{\theta}} \equiv 0 .
$$

Thus, under the dynamics of (76) yields:

$$
\begin{aligned}
\dot{x}_{p}= & -M_{d} M^{-1} \nabla_{x_{q}} H_{x}+\left(J_{2}-R_{d}\right) \nabla_{x_{p}} H_{x}+\Lambda \nabla_{x_{\tilde{\theta}}} H_{x}=0 \\
= & -M_{d} M^{-1}(\nabla_{x_{q}} V_{x}+\underbrace{\frac{1}{2} x_{p}^{\top}\left(\nabla_{x_{q}} M_{d}^{-1}\right) x_{p}}_{=0}) \\
& +\underbrace{\left(J_{2}-R_{d}\right) M_{d}^{-1} x_{p}}_{=0}+\underbrace{\Lambda \tilde{\theta}}_{=0}=0 \\
= & -M_{d} M^{-1} \nabla_{x_{q}} V_{x}=0 .
\end{aligned}
$$

This implies $\nabla_{x_{q}} V_{x}=0$. Invoking similar arguments to those in the proof of Proposition 3.2, we obtain

$$
\nabla_{x_{q}} V_{x} \equiv 0 \Longrightarrow x_{q}=q_{e}
$$

Since all trajectories converge to their desired values, we can conclude that the closed-loop system (51) has an asymptotically stable equilibrium at $\left(q_{e}, 0, \theta\right)$. 
The controller is obtained by matching the momenta of (1) and (76), that is

$$
\begin{aligned}
\dot{p} \equiv & \dot{x}_{p}+\mathcal{U} \dot{\hat{\theta}} \\
G u & -\nabla_{q} H=-M_{d} M^{-1} \nabla_{x_{q}} H_{x}+\left(J_{2}-R_{d}\right) \nabla_{x_{p}} H_{x} \\
& +\Lambda \nabla_{x_{\tilde{\theta}}} H_{x}+\mathcal{U} \dot{\hat{\theta}} \\
G u & -F \theta-\frac{1}{2} \sum_{i=1}^{n} e_{i} p^{\top} \nabla_{q_{i}} M^{-1} p=-M_{d} M^{-1}(F \theta+S) \\
& \quad-\frac{1}{2} M_{d} M^{-1} \sum_{i=1}^{n} e_{i} x_{p}^{\top} \nabla_{q_{i}} M_{d}^{-1} x_{p} \\
& +\left(J_{2}-R_{d}\right) M_{d}^{-1} x_{p}+\Lambda \tilde{\theta}+\mathcal{U} \dot{\hat{\theta}} .
\end{aligned}
$$

Substituting $x_{p}=p-\mathcal{U} \tilde{\theta}$ and (77) in (83) yields:

$$
\begin{aligned}
G u= & F \theta+\frac{1}{2} \sum_{i=1}^{n} e_{i} p^{\top} \nabla_{q_{i}} M^{-1} p-M_{d} M^{-1}(F \theta+S) \\
& -\frac{1}{2} M_{d} M^{-1} \sum_{i=1}^{n} e_{i} p^{\top} \nabla_{q_{i}} M_{d}^{-1} p \\
& +M_{d} M^{-1} \sum_{i=1}^{n} e_{i} p^{\top} \nabla_{q_{i}} M_{d}^{-1} \mathcal{U} \tilde{\theta} \\
& -\frac{1}{2} M_{d} M^{-1} \sum_{i=1}^{n} e_{i} \mathcal{U} \tilde{\theta}^{\top} \nabla_{q_{i}} M_{d}^{-1} \mathcal{U} \tilde{\theta} \\
& +\left(F-M_{d} M^{-1} F+\left(J_{2}-R_{d}\right) M_{d}^{-1} \mathcal{U}\right. \\
& -M_{d} M^{-1} \sum_{i=1}^{n} e_{i} p^{\top} \nabla_{q_{i}} M_{d}^{-1} \mathcal{U} \\
& \left.+\frac{1}{2} M_{d} M^{-1} \sum_{i=1}^{n} e_{i} \mathcal{U} \tilde{\theta}^{\top} \nabla_{q_{i}} M_{d}^{-1} \mathcal{U}\right) \tilde{\theta} \\
& +\left(J_{2}-R_{d}\right) M_{d}^{-1} p-\left(J_{2}-R_{d}\right) M_{d}^{-1} \mathcal{U} \tilde{\theta}+\mathcal{U} \dot{\hat{\theta}} .
\end{aligned}
$$

Using $\tilde{\theta}=\hat{\theta}-\theta$ and canceling like terms but with opposite signs, we obtain the controller (80). Finally, the update law is computed as:

$$
\begin{aligned}
\dot{x}_{\hat{\theta}}= & -M^{-1} \mathcal{U} \nabla_{x_{q}} H_{x}-\Lambda \nabla_{x_{p}} H_{x}-\Upsilon \nabla_{x_{\tilde{\theta}}} H_{x} \\
= & -M^{-1} \mathcal{U}(F \theta+S)-\frac{1}{2} M^{-1} \mathcal{U} \sum_{i=1}^{n} e_{i} x_{p}^{\top} \nabla_{q_{i}} M_{d}^{-1} x_{p} \\
& -\Lambda M_{d}^{-1} x_{p}-\Upsilon \tilde{\theta} .
\end{aligned}
$$

Substituting $x_{p}=p-\mathcal{U} \tilde{\theta}$, (77) and (78) in (84), and using $\tilde{\theta}=\hat{\theta}-\theta$ then canceling like terms but with opposite signs, we obtain the update law (81). Hence, it completes the proof.

\section{APPLICATION: THE INERTIA WHEEL PENDULUM}

We use the Quanser IWP module [21], whose simplified free body diagram of the mechanical part is shown in Figure 1. It consists of an unactuated planar inverted pendulum with an actuated symmetric disk/wheel attached to its end, which is free to rotate about an axis parallel to the axis of rotation of the pendulum. The system has two degrees-of-freedom; the angular position of the pendulum $q_{1}$ and the angular position of the wheel $q_{2}$. Only the wheel is actuated by a motor, hence the system is underactuated. The dynamic equations of the
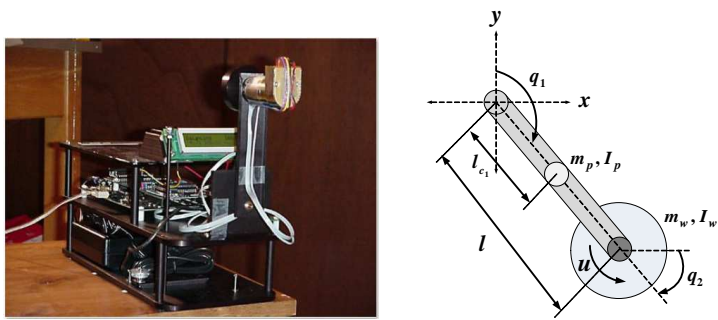

Fig. 1: The Inertia Wheel Pendulum.

IWP system can be written in a PCH form (1) with $n=2$, $m=1$ and

$$
\begin{gathered}
M=\left[\begin{array}{ll}
k_{1} & k_{2} \\
k_{2} & k_{2}
\end{array}\right], G=e_{2}=\left[\begin{array}{l}
0 \\
1
\end{array}\right], \text { and } \\
V\left(q_{1}\right)=k_{3}\left(1+\cos \left(q_{1}\right)\right),
\end{gathered}
$$

where, the control input $u$ is the motor torque, $k_{1}=m_{p} l_{c_{1}}^{2}+$ $m_{w} l^{2}+I_{p}+I_{w}, k_{2}=I_{w}$ and $k_{3}=g\left(m_{p} l_{c_{1}}+m_{w} l\right)$. The values of the model parameters are as follows [21]): $m_{p}=0.2164$, $m_{w}=0.085, l=0.2346, l_{c_{1}}=0.1173, I_{p}=2.233 \times 10^{-4}$, $I_{w}=2.495 \times 10^{-5}$, and $g=9.81$.

\section{A. IDA-PBC Stabilizing Controller}

To start with, a stabilizing controller is obtained using IDA$\mathrm{PBC}$ design procedures proposed in [9]. The main objective is to provide a continuous control law to swing up the pendulum by spinning the wheel and to stabilize it at its upward position $q=\left(0, q_{2}\right)$ for any $q_{2} \in[0,2 \pi]$. By fixing $M_{d}$ to be a constant matrix of the form

$$
M_{d}=\Delta\left[\begin{array}{ll}
m_{1} & m_{2} \\
m_{2} & m_{3}
\end{array}\right]=\Delta\left[\begin{array}{cc}
m_{1} & \left(\frac{k_{2}}{k_{1}}\right) m_{1}+\varepsilon \\
\left(\frac{k_{2}}{k_{1}}\right) m_{1}+\varepsilon & m_{3}
\end{array}\right],
$$

where $\varepsilon>0, \Delta=k_{1} k_{2}-k_{2}^{2}$ and having $G^{\perp}=\left[\begin{array}{ll}1 & 0\end{array}\right]$, the desired Hamiltonian (4) is obtained as

$$
\begin{gathered}
H_{d}=\frac{1}{2} p^{\top} M_{d}^{-1} p+V_{d}(q), \\
V_{d}(q)=-k_{3} \gamma_{1} \cos \left(q_{1}\right)+\frac{1}{2} K_{p}\left(\varepsilon k_{1} \gamma_{1} q_{1}+q_{2}\right)^{2},
\end{gathered}
$$

with $\gamma_{1}=\frac{1}{k_{2}\left(m_{2}-m_{1}\right)}$ and $K_{p}>0$ the gain of the energy shaping controller which is calculated as

$$
u_{e s}=\gamma_{2} \sin \left(q_{1}\right)+K_{p} \gamma_{3}\left(\varepsilon k_{1} \gamma_{1} q_{1}+q_{2}\right),
$$

with $\gamma_{2}=-k_{3} \gamma_{1}\left(m_{2} k_{2}-m_{3} k_{2}\right), \gamma_{3}=-\varepsilon k_{1} \gamma_{1}\left(m_{2} k_{2}-\right.$ $\left.m_{3} k_{2}\right)-\left(-m_{2} k_{2}+m_{3} k_{1}\right)$. The damping injection controller is

$$
u_{d i}=-K_{v} \frac{\Delta}{\Delta_{d}}\left(-m_{2} p_{1}+m_{1} p_{2}\right),
$$

with $\Delta_{d}=\operatorname{det}\left(M_{d}\right)=\Delta^{2}\left(m_{1} m_{3}-m_{2}^{2}\right)$ and $K_{v}>0$ the damping injection controller gain. 


\section{B. Integral Action Controller}

We apply the procedure given in Proposition 3.4 to design the integral controller for the IWP system. Given $G=$ $\left[\begin{array}{ll}0 & 1\end{array}\right]^{\top}$, then the matrix $\mathcal{G}$ is defined as

$$
\mathcal{G}=\left[\begin{array}{ll}
0 & 0 \\
0 & 1
\end{array}\right]
$$

The IC on the non-passive output is then calculated as

$$
\begin{aligned}
v & =K_{v} K_{i} \frac{\Delta}{\Delta_{d}}\left(m_{2} k_{1}-m_{1} k_{2}\right) x_{v}, \\
\dot{x}_{v} & =K_{p}\left(\varepsilon k_{1} \gamma_{1} q_{1}+q_{2}\right)
\end{aligned}
$$

and the extra term $u_{p}=-K_{i} k_{2} K_{p}\left(\varepsilon k_{1} \gamma_{1} q_{1}+q_{2}\right)$.

\section{ISS Controller}

Following the ISS controller design presented in Subsection [III-D in particular Proposition 3.5, the control input is obtained as

$$
\begin{aligned}
u & =u_{I D A}-\frac{\rho \Delta}{\Delta_{d}}\left(\beta_{1}\left(m_{3} p_{1}-m_{2} p_{2}\right)-\beta_{2}\left(m_{2} p_{1}-m_{1} p_{2}\right)\right) \\
& -\frac{\rho \Delta}{\Delta_{d}}\left(\beta_{1}\left(m_{3} k_{2}-m_{2} k_{2}\right)-\beta_{2}\left(m_{2} k_{2}-m_{1} k_{2}\right)\right) x_{v} \\
& -K_{i} K_{v} k_{2} \frac{\Delta}{\Delta_{d}}\left(m_{2}+m_{1}\right) x_{v}-K_{i} k_{2} K_{p}\left(\varepsilon k_{1} \gamma_{1} q_{1}+q_{2}\right),
\end{aligned}
$$

with the update law

$$
\dot{x}_{v}=K_{i} K_{p}\left(\varepsilon k_{1} \gamma_{1} q_{1}+q_{2}\right),
$$

and

$$
\begin{aligned}
\beta_{1}= & K_{p}\left(\varepsilon k_{1} \gamma_{1} q_{1}+q_{2}\right)\left(k_{3} \gamma_{1} \sin \left(q_{1}\right)\right. \\
& \left.+K_{p} \varepsilon k_{1} \gamma_{1}\left(\varepsilon k_{1} \gamma_{1} q_{1}+q_{2}\right)\right) \\
\beta_{2}= & K_{p}^{2}\left(\varepsilon k_{1} \gamma_{1} q_{1}+q_{2}\right)^{2} .
\end{aligned}
$$

\section{Adaptive-IDA-PBC Controller}

Following the discussion in Subsection $\amalg I-E$ here we show the design of an adaptive controller to compensate for the uncertainty in the potential energy function $V(q)$. Consider the potential energy function of IWP system (86), the gradient of this function is

$$
\nabla_{q} V=-k_{3} \sin \left(q_{1}\right),
$$

which can be linearly parametrized as (41), with $F\left(q_{1}\right)=$ $-\sin \left(q_{1}\right)$ and $\theta=k_{3}$ the uncertain term. Thus, the energy shaping controller 90) is rewritten as

$$
u_{e s}=-\theta \gamma_{1}\left(m_{2} k_{2}-m_{3} k_{2}\right) \sin \left(q_{1}\right)+K_{p} \gamma_{3}\left(\varepsilon k_{1} \gamma_{1} q_{1}+q_{2}\right) .
$$

Thus, the overall adaptive-IDA control input is obtained as

$$
\begin{aligned}
u= & -\hat{\theta} \gamma_{1}\left(m_{2} k_{2}-m_{3} k_{2}\right) \sin \left(q_{1}\right)+K_{p} \gamma_{3}\left(\varepsilon k_{1} \gamma_{1} q_{1}+q_{2}\right) \\
& -K_{v} \frac{\Delta}{\Delta_{d}}\left(-m_{2} p_{1}+m_{1} p_{2}\right) \\
\dot{\hat{\theta}}= & -\frac{\Delta}{\Delta_{d}}\left(k_{2} m_{1}-k_{2} m_{2}-1\right)\left(-m_{3} p_{1}+m_{2} p_{2}\right) \hat{\theta} \sin \left(q_{1}\right) .
\end{aligned}
$$

Fig. 3: Angle of the wheel $q_{2}$ for the tracking and disturbance rejection control problem.

2) ISS simulations: The ISS control law described above
ave been implemented on the IWP system for both matched
unmatched disturbances cases with the design parameters

2) ISS simulations: The ISS control law described above
have been implemented on the IWP system for both matched
and unmatched disturbances cases with the design parameters

2) ISS simulations: The ISS control law described above
have been implemented on the IWP system for both matched
and unmatched disturbances cases with the design parameters

\section{E. Simulations}

In this section, the integral, ISS and adaptive IDA controllers designed for the IWP system are implemented in a MATLAB/SIMULINK environment to evaluate the performance of the control system. In all simulations, the initial condition $\left[q_{0}, p_{0}\right]=[\pi, 0,0,0]$ for the system is used.

1) Integral IDA-PBC simulations: Here, we show simulations for a tracking control problem where the pendulum is required to track a sinusoidal reference signal $q_{1_{r}}$. A constant force disturbance of $1 N$ is also injected into the dynamic of $q_{1}$. We implement the integral IDA-PBC controller with the parameters $m_{1}=0.4, m_{3}=5, \epsilon=1, K_{p}=0.5$, $K_{v}=1 \times 10^{-5}$ and $K_{i}=1.2$. The simulation results in Figure 2 show that without integral action, the system subject to external disturbance exhibits a large steady-state error, which can be observed particularly in the trajectory of $q_{2}$. With integral action, the trajectories track their desired references despite the presence of the constant disturbance, bringing the trajectories to converge smoothly to their desired values. In Figure 3 we show the trajectory of $q_{2}$ comparing it to the case of no disturbance as the reference.
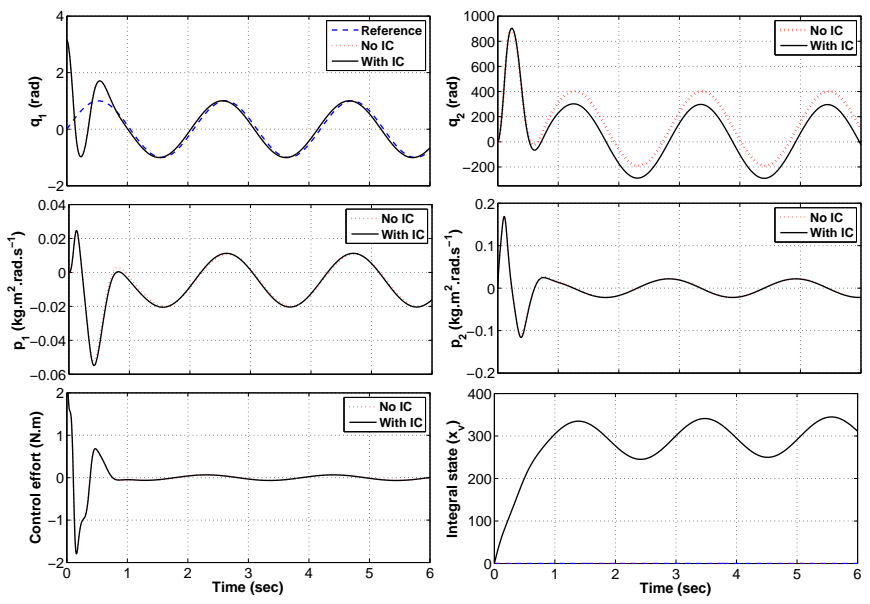

Fig. 2: State trajectories and control input of the IWP system for the tracking and disturbance rejection control problem.

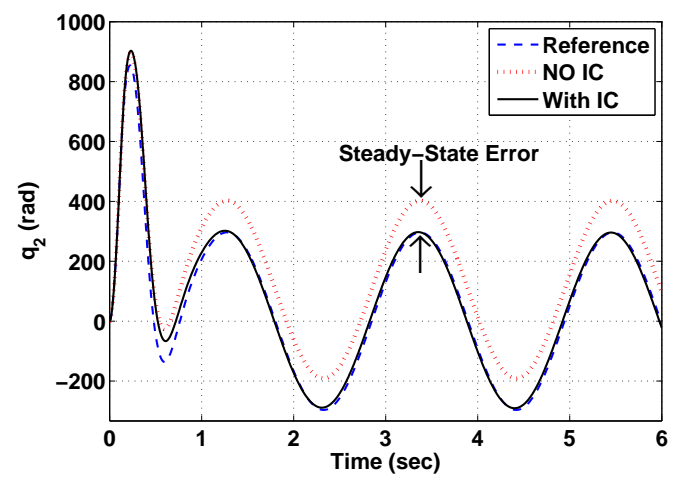


$m_{1}=0.4, m_{3}=5, \epsilon=1, K_{p}=1.1$, and $K_{v}=5.6 \times 10^{-5}$. The disturbance vector is selected as $d=\lambda \tanh (\dot{p})$.

We first consider the robust control problem of the IWP system having matched disturbances. Figures 4 and 5 show the behaviour of the system subject to small $(\lambda=0.5)$ and large $(\lambda=1)$ disturbances where the ISS controller parameters have been chosen as $K_{i}=1$ and $\rho=0.1 \times 10^{-12}$. As expected, we can see that all states (signals) converge to their respective desired equilibrium values with high performance.
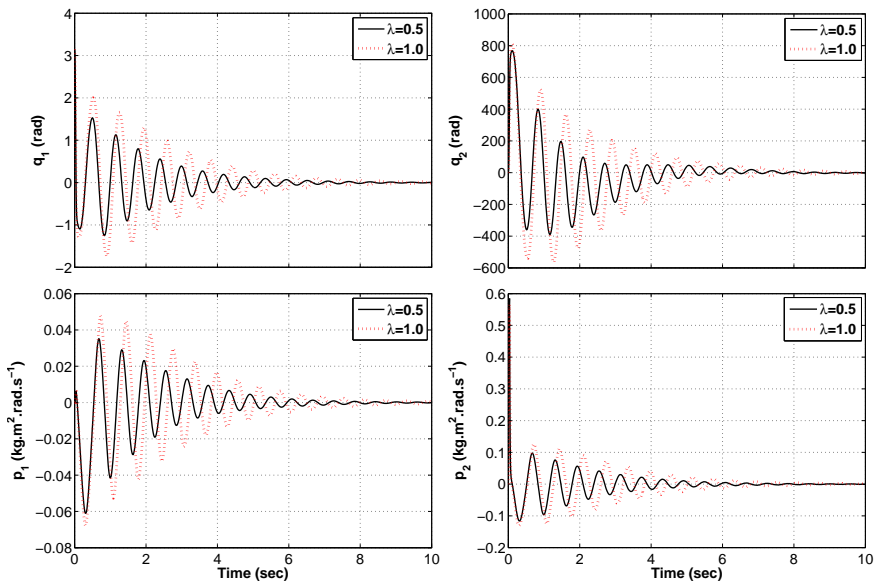

Fig. 4: State trajectories of the IWP system for matched disturbance control problem.
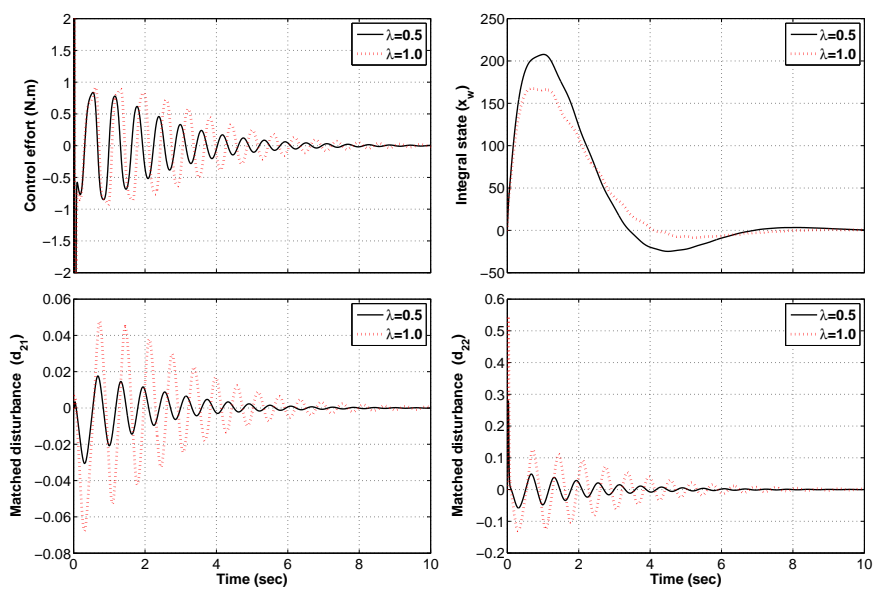

Fig. 5: Control input, update law and disturbance inputs of the IWP for matched disturbance control problem.

For the case of unmatched disturbances, we have selected two different sets of controller's parameters $\left(K_{p}=1.1, K_{v}=\right.$ $\left.5.6 \times 10^{-5}, K_{i 1}=1.5, \rho_{1}=0.09 \times 10^{-12}\right)$ and $\left(K_{p}=\right.$ $\left.0.4, K_{v}=5.6 \times 10^{-5}, K_{i 2}=1.5, \rho_{2}=0.09 \times 10^{-11}\right)$, in response to two different disturbance gains $(\lambda=60)$ and $(\lambda=90)$, respectively. The simulation results are shown in Figures 6 and 7 Again, we can see the convergence of all states to their desired values with reasonable transients. These figures also shows that for relatively high disturbances $(\lambda=90)$, we have selected a large value of $\rho_{2}$ to enlarge the domain of attraction and thus the system is ultimately bounded. This follows the proof of Proposition 3.5 Notice that we have also decreased the proportional gain $K_{p}$ to make sure that the maximum torque does not exceed the actuator limit.
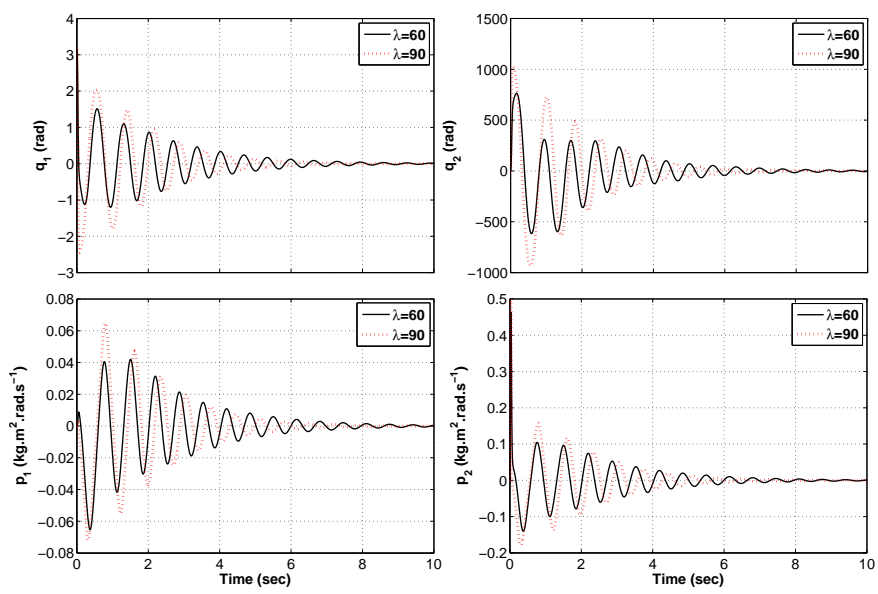

Fig. 6: State trajectories of the IWP system for unmatched disturbance control problem.
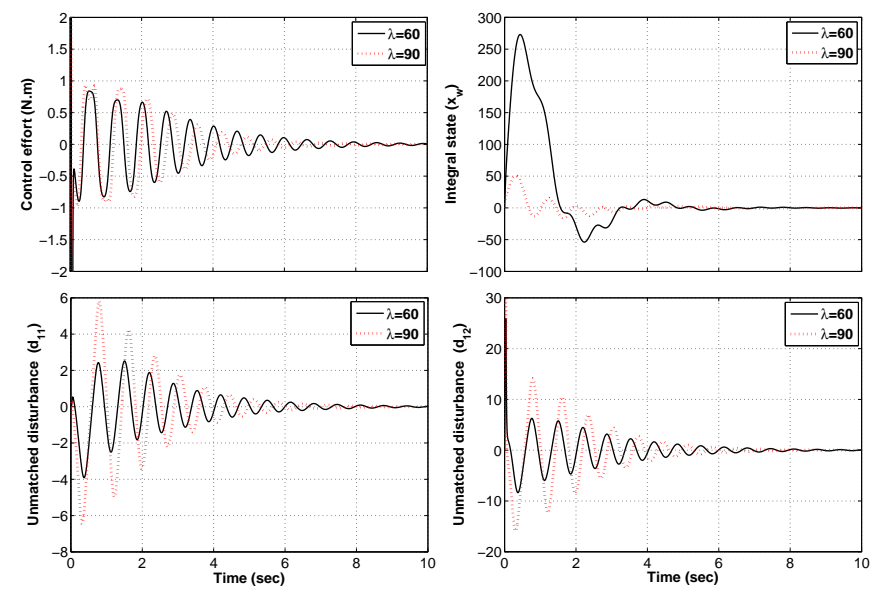

Fig. 7: Control input, update law and disturbance input of the IWP for unmatched disturbance control problem.

3) Adaptive IDA-PBC simulations: For the adaptive control method, we have selected the parameters and gains of the controller as $m_{1}=0.2, m_{3}=10, \epsilon=1, K_{p}=4.5$, and $K_{v}=2.2 \times 10^{-4}$. Furthermore, we have adjusted the uncertain term $\theta=k_{3}$ as $\theta=\vartheta+\zeta$, with $\zeta$ is a fixed estimate. This enables us to compare this method with the non-adaptive one. Given the value of $\theta=g\left(m_{p} l_{c_{1}}+m_{w} l\right)$, we have selected $\zeta=\theta / 2$ for this case.

Figures 8 and 9 show a comparative plot of the system's response with the adaptive IDA controller and the non-adaptive IDA-PBC controller. As shown, without adaptation law the uncertainty in $V(q)$ results in a relatively large steady state error and unacceptable transients. In contrast, adding the proposed adaptive law, the trajectories of the IWP system converge to their desired states with excellent performance. Figure 9 shows the convergence of the estimate $\hat{\theta}$ to the true value $\theta$

\section{CONCLUSION}

In this paper, we have presented several control designs to deal with several robustness-related issues within $\mathrm{PCH}$ 

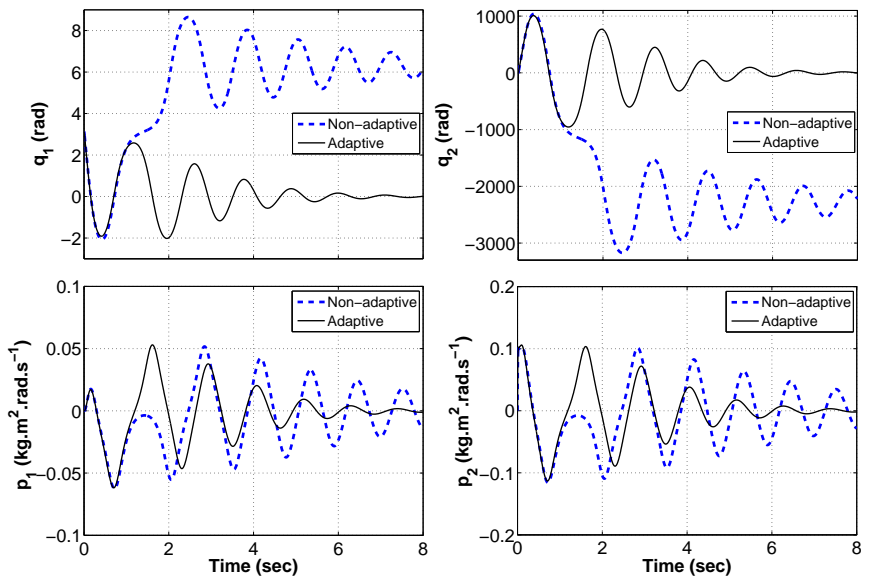

Fig. 8: State trajectories of the IWP system for the adaptive control problem.
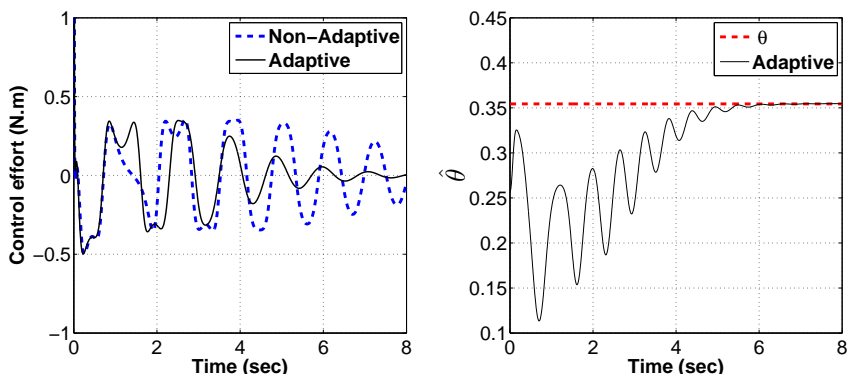

Fig. 9: Control input and update law (estimate) of the IWP system for the adaptive control problem.

framework. In particular, IDA-PBC method along with a dynamic state-feedback controller that involves integral action is used to improve the robustness of the closed-loop system. First, we have presented several results on IC for a class of $\mathrm{PCH}$ systems, extending the results of [6], [7]. Second, we have provided a general framework that allows the use of integral action for underactuated mechanical systems. This work is the first that discusses the incorporation of IC for underactuated mechanical system within PCH framework. The matched and unmatched disturbance rejection problems are proved using the integral action controller with a particular change of coordinates that involves adding some damping terms. These results ensure that the ISS property is satisfied and can prove, for perturbations that satisfy some conditions, that asymptotic stability of the desired equilibrium can be also achieved. An initial adaptive framework to deal with parametric uncertainties in $\mathrm{PCH}$ models, in particular uncertainties in the potential energy function, has been also presented.

Application to an inertia wheel pendulum which is an underactuated system has been presented, and the effectiveness of the proposed controllers has been shown through numerical simulations. The simulation results demonstrate that the system is robust with respect to different perturbations, preserving the $\mathrm{PCH}$ structure, retaining the (asymptotic) stability with high performance. While only one example is presented as illustration, other $\mathrm{PCH}$ systems belong to class (see for instance [8]) are possible systems to apply our results.

\section{REFERENCES}

[1] R. Ortega and E. Garcia-Canseco, "Interconnection and damping assignment passivity-based control: A survey," European Journal of Control, vol. 10, pp. 432-450, 2004.

[2] H. Khalil, Nonlinear Systems, 3rd Ed. Prentice Hall, 2002.

[3] R. Ortega, A. van der Schaft, B. Maschke, and G. Escobar, "Interconnection \& damping assignment passivity-based control of port-controlled Hamiltonian systems," Automatica, vol. 38, pp. 585-596, 2002.

[4] R. Ortega, A. Van Der Schaft, I. Mareels, and B. Maschke, "Putting energy back in control," Control Systems, IEEE, vol. 21, no. 2, pp. 1833, 2001.

[5] A. van der Schaft, L2-Gain and Passivity Techniques in Nonlinear Control. Berlin Heidelberg: Springer, 2000.

[6] A. Donaire and S. Junco, "On the addition of integral action to portcontrolled hamiltonian systems," Automatica, vol. 45, no. 8, pp. $1910-$ 1916, 2009.

[7] J. G. Romero, A. Donaire, and R. Ortega, "Robust energy shaping control of mechanical systems," Systems \& Control Letters, vol. 62, no. 9, pp. $770-780,2013$

[8] Y. Liu and H. Yu, "A survey of underactuated mechanical systems," IET Control Theory and Applications, vol. 7, pp. 921-935, 2013.

[9] M. Ryalat and D. S. Laila, "IDA-PBC for a Class of Underactuated Mechanical Systems with Application to a Rotary Inverted Pendulum," in 52nd IEEE Conference on Decision and Control, 2013, pp. 52405245.

[10] K. Fujimoto, K. Sakurama, and T. Sugie, "Trajectory tracking control of port-controlled hamiltonian systems via generalized canonical transformations," Automatica, vol. 39, no. 12, pp. 2059 - 2069, 2003.

[11] R. Ortega and J. G. Romero, "Robust integral control of port-hamiltonian systems: The case of non-passive outputs with unmatched disturbances," Systems \& Control Letters, vol. 61, no. 1, pp. 11 - 17, 2012.

[12] R. Ortega, M. Spong, F. Gomez-Estern, and G. Blankenstein, "Stabilization of a class of underactuated mechanical systems via interconnection and damping assignment," Automatic Control, IEEE Transactions on, vol. 47, pp. 1218 - 1233, 2002.

[13] J. A. Acosta, R. Ortega, A. Astolfi, and A. D. Mahindrakar, "Interconnection and damping assignment passivity based control of mechanical systems with underactuation degree one," IEEE Trans. Auto. Control, vol. 50, pp. 1936-1955, 2005.

[14] F. Gmez-Estern and A. V. der Schaft, "Physical damping in ida-pbc controlled underactuated mechanical systems," European Journal of Control, vol. 10, no. 5, pp. 451 - 468, 2004.

[15] I. Sarras, R. Ortega, and A. J. van der Schaft, "On the modeling, linearization and energy shaping control of mechanical systems," in 4th IFAC Workshop on Lagrangian and Hamiltonian Methods for Non Linear Control, 2012, pp. 161-166.

[16] K. Zhou and J. C. Doyle, Essential of Robust Control. New Jersey: Prentice Hall, 1998.

[17] E. Sontag, "Smooth stabilization implies coprime factorization," IEEE Transactions on Automatic Control, vol. 34, no. 4, pp. 435-443, Apr 1989.

[18] - "Input to state stability: Basic concepts and results," in Nonlinear and Optimal Control Theory, P. Nistri and G. Stefani, Eds. Springer Berlin Heidelberg, 2008, pp. 163-220.

[19] E. Sontag and Y. Wang, "On characterizations of the input-to-state stability property," Systems \& Control Letters, vol. 24, no. 5, pp. 351359,1995

[20] A. Astolfi, D. Karagiannis, and R. Ortega, Nonlinear and adaptive control design and applications. London: Springer-Verlag, 2007.

[21] D. Block, K. Astrom, and M. Spong, The Reaction Wheel Pendulum. Morgan \& Claypool Publishers, 2007. 